\title{
Torque-saturated, Inertia-free Spacecraft Attitude Control
}

\author{
Gerardo Cruz, Xuebo Yang ${ }^{\dagger}$ Avishai Weiss \\ Ilya Kolmanovsky§, Dennis S. Bernstein $₫$ \\ University of Michigan, 1320 Beal Ave., Ann Arbor, MI 48109
}

July 18, 2011

\begin{abstract}
Torque-saturated control laws for spacecraft attitude control are considered. These control laws require no knowledge of the mass distribution of the spacecraft and thus are inertia-free. We examine two specific problems. In the first problem, the spacecraft has an arbitrary initial attitude and angular velocity, and the objective is to bring the spacecraft to rest with a specified attitude. In the second problem, the spacecraft has an arbitrary initial attitude and angular velocity, and the objective is to bring the spacecraft to a spin about a specified body axis with a specified attitude. The body axis need not be a principal axis. Both problems are considered under the assumption that the torque, which is continuously variable, is limited by a saturation constraint. Disturbances are assumed to be zero.
\end{abstract}

\footnotetext{
*Graduate Student, Department of Aerospace Engineering

${ }^{\dagger}$ Graduate Student, Department of Aerospace Engineering

${ }^{\ddagger}$ Graduate Student, Department of Aerospace Engineering

$\S$ Professor, Department of Aerospace Engineering

ฯProfessor, Department of Aerospace Engineering
} 


\section{Introduction}

Control of spacecraft remains a challenging problem due to nonlinearities, uncertainties, and hardware constraints. The starting point for the present paper is [1], which provides attitude-tracking control laws for rigid spacecraft in the presence of unmeasured harmonic disturbances. The control law in [1] requires knowledge of the spectrum of the disturbance, but no knowledge of the spacecraft's mass distribution. This control law is thus inertia-free.

The actuation considered in [1] is based on continuously variable torques without momentum storage and without a bound on the torque magnitude. The assumption of zero on-board momentum storage is removed in [2], where the results of [1] are extended to the case of reaction wheel actuation. However, torque limits are not considered in [2]. Constraints on torque magnitude for inertia-dependent control laws are treated in $[13-15]$.

The goal of the present paper is to extend the results of [1] by modifying the attitude-tracking control laws to satisfy a constraint on the torque magnitude. We consider two specific problems. In the first problem, the spacecraft has an arbitrary initial attitude and angular velocity, and the objective is to bring the spacecraft to rest with a specified attitude. We call this problem motion to rest (M2R). In the second problem, the spacecraft has an arbitrary initial attitude and angular velocity, and the objective is to bring the spacecraft to a spin about a specified body axis and with a specified attitude. We call this problem motion to spin (M2S). The body axis need not be a principal axis. Both problems are considered under the assumption that the torque, which is continuously variable, is limited by a saturation constraint.

For the M2R problem, the controller developed in [1] is a static feedback control law that acts directly on measurements of attitude and angular velocity. For the M2S problem, the controller is a dynamic a 6 th-order feedback compensator that uses on-line estimates of the spacecraft inertia. This control law does not depend on convergence of the inertia estimates to the true estimates. Thus, persistency of excitation conditions are not needed to guarantee tracking. In the absence of a torque-magnitude constraint, these control laws are shown in [1] to provide almost global stabilization, that is, convergence to the specified equilibrium except for a set of initial conditions of measure zero. In order to avoid the singularities of Euler angles and Rodrigues parameters, as well as the switching discontinuity required by quaternions to achieve global convergence [3-7], these control laws use rotation matrices to parameterize the orthogonal group $\mathrm{SO}(3)[10-12]$.

In the present paper, the M2R and M2S control laws are modified to account for a saturation constraint on the magnitude of the torque. For the M2R control law, this modification is shown to provide almost global stabilization. For the M2S control law, the modification is ad hoc, but numerical investigation of the Lyapunov derivative for the case of unconstrained torque suggests that stability is preserved.

The performance of the M2R and M2S control laws is studied numerically. In particular, we vary the torque-saturation level, and show that the performance degrades gracefully as the torque-saturation level decreases. For M2S, we consider commanded spins about minor, intermediate, and major principal axes, and we show that these spins are achievable under arbitrarily small saturation levels. Although the ability to perform such spins under arbitrary torque-saturation levels may be intuitively expected, we are not aware of any control laws that guarantee this ability. For spins about non-principal axes, we investigate the degradation of the M2S control law as the saturation magnitude decreases to a level below the required steady-state torque predicted by Euler's equation. 


\section{Spacecraft Model}

As a spacecraft model, we consider a single rigid body controlled by force or torque actuators, such as thrusters or magnetic torque devices, and without on-board momentum storage. We consider only the rotational motion of the spacecraft and not the translational motion of the spacecraft's center of mass; therefore we consider only the torque applied by the force actuators. We assume that a body-fixed frame is defined for the spacecraft, whose origin is at the spacecraft's center of mass, and that an inertial frame is specified for determining the attitude of the spacecraft. The spacecraft equations of motion are given by Euler's equation and Poisson's equation, which are, respectively,

$$
\begin{aligned}
J \dot{\omega} & =(J \omega) \times \omega+B u+z_{d}, \\
\dot{R} & =R \omega^{\times},
\end{aligned}
$$

where $\omega \in \mathbb{R}^{3}$ is the angular velocity of the spacecraft frame with respect to the inertial frame resolved in the spacecraft frame, $\omega^{\times}$is the skew-symmetric cross-product matrix of $\omega, J \in \mathbb{R}^{3 \times 3}$ is the constant, positivedefinite inertia matrix of the spacecraft, that is, the inertia dyadic of the spacecraft relative to its center of mass resolved in the spacecraft frame, and $R \in \mathbb{R}^{3 \times 3}$ is the proper orthogonal matrix (that is, rotation matrix) that transforms the components of a vector resolved in the spacecraft frame into the components of the same vector resolved in the inertial frame.

Since $J$ is an inertia matrix, its eigenvalues $\lambda_{1}, \lambda_{2}, \lambda_{3}$ are real and satisfy the triangle rule. That is, $\lambda_{1}<\lambda_{2}+\lambda_{3}$, where $0<\lambda_{3} \leq \lambda_{2} \leq \lambda_{1}$. The components of the vector $u \in \mathbb{R}^{3}$ represent three independent torque inputs, while the matrix $B \in \mathbb{R}^{3 \times 3}$ determines the applied torque about each axis of the spacecraft frame due to $u$ as given by the product $B u$. The vector $z_{d}$ represents disturbance torques, that is, all internal and external torques applied to the spacecraft aside from control torques, which may be due to onboard components, gravity gradients, solar pressure, atmospheric drag, or the ambient magnetic field. For convenience in (1), (2) we omit the argument $t$, recognizing that $\omega, R, u$ and $z_{d}$ are time-varying quantities.

Both rate (inertial) and attitude (noninertial) measurements are assumed to be available. Gyro measurements $y_{\text {rate }} \in \mathbb{R}^{3}$ are assumed to provide measurements of the angular velocity resolved in the spacecraft frame, that is,

$$
y_{\text {rate }}=\omega .
$$

We assume that gyro measurements are available without noise and without bias. In practice, bias can be corrected by using attitude measurements.

Attitude is measured indirectly through direction measurements using sensors such as star trackers. The attitude is determined as

$$
y_{\text {attitude }}=R \text {. }
$$

The objective of the attitude control problem is to determine control inputs such that the spacecraft attitude given by $R$ follows a commanded attitude trajectory given by a possibly time-varying $\mathrm{C}^{1}$ rotation matrix $R_{\mathrm{d}}(t)$. For $t \geq 0, R_{\mathrm{d}}(t)$ is given by

$$
\begin{aligned}
\dot{R}_{\mathrm{d}}(t) & =R_{\mathrm{d}}(t) \omega_{\mathrm{d}}(t)^{\times}, \\
R_{\mathrm{d}}(0) & =R_{\mathrm{d} 0},
\end{aligned}
$$

where $\omega_{\mathrm{d}}$ is the desired and possibly time-varying angular velocity. The error between $R(t)$ and $R_{\mathrm{d}}(t)$ is given in terms of the attitude-error rotation matrix

$$
\tilde{R} \triangleq R_{\mathrm{d}}^{\mathrm{T}} R,
$$


which satisfies the differential equation

$$
\dot{\tilde{R}}=\tilde{R} \tilde{\omega}^{\times}
$$

where the angular velocity error $\tilde{\omega}$ is defined by

$$
\tilde{\omega} \triangleq \omega-\tilde{R}^{\mathrm{T}} \omega_{\mathrm{d}}
$$

We rewrite (1) in terms of the angular-velocity error as

$$
J \dot{\tilde{\omega}}=\left[J\left(\tilde{\omega}+\tilde{R}^{\mathrm{T}} \omega_{\mathrm{d}}\right)\right] \times\left(\tilde{\omega}+\tilde{R}^{\mathrm{T}} \omega_{\mathrm{d}}\right)+J\left(\tilde{\omega} \times \tilde{R}^{\mathrm{T}} \omega_{\mathrm{d}}-\tilde{R}^{\mathrm{T}} \dot{\omega}_{\mathrm{d}}\right)+B u+z_{d} .
$$

A scalar measure of attitude error is given by the rotation angle $\theta(t)$ about an eigenaxis needed to rotate the spacecraft from its attitude $R(t)$ to the desired attitude $R_{\mathrm{d}}(t)$ [16], i.e.,

$$
\theta(t)=\cos ^{-1}\left(\frac{1}{2}[\operatorname{tr} \tilde{R}(t)-1]\right) .
$$

We now summarize the assumptions upon which the following development is based:

Assumption 1. $J$ is constant but unknown.

Assumption 2. $B$ is constant, nonsingular, and known.

\section{Motion-to-Rest (M2R) Control Law}

We now consider the objective of bringing the spacecraft from an arbitrary initial attitude and angular velocity to rest with a specified attitude. Thus, we assume that the desired attitude $R_{\mathrm{d}}$ is constant and $\omega_{\mathrm{d}}=0$. We also assume that the disturbance $z_{d}$ is zero. In this case, we consider the Lyapunov function candidate

$$
V(\omega, \tilde{R}) \triangleq \frac{1}{2} \omega^{\mathrm{T}} J \omega+K_{\mathrm{p}} \operatorname{tr}(A-A \tilde{R}) .
$$

The proportional-derivative-(PD)-type control law

$$
u=-B^{-1}\left(K_{\mathrm{p}} S+K_{\mathrm{v}} \omega\right),
$$

where $K_{\mathrm{p}}$ is a positive scalar, $K_{\mathrm{v}}(\omega) \in \mathbb{R}^{3 \times 3}$ for each $\omega \in \mathbb{R}^{3}$ is positive definite, and

$$
S \triangleq \sum_{i=1}^{3} a_{i}\left(\tilde{R}^{\mathrm{T}} e_{i}\right) \times e_{i}
$$

where $a_{i}>0$ for $i=1,2,3$ are the distinct diagonal entries of the diagonal matrix $A$, yields

$$
\dot{V}(\omega, \tilde{R})=-\omega^{\mathrm{T}} K_{\mathrm{v}} \omega .
$$

As shown in [8], (14) implies almost global stabilization of the constant desired attitude $R_{\mathrm{d}}$. This control law achieves zero steady-state error for constant-attitude setpoint commands without integral action and without knowledge of $J$.

The interpretation of the gains $K_{v}$ and $K_{p}$ as rate and position gains is useful in suggesting how their values can be adjusted to tune the dynamics of the closed-loop system. For further discussion, see [10]. 
The following result shows that, for slew maneuvers without disturbances, it is possible to arbitrarily bound the level of torque about each axis. Let $\sigma_{\max }(M)$ and $\sigma_{\min }(M)$ denote, respectively, the maximum and minimum singular values of the matrix $M$. Furthermore, let $\|x\|_{\infty}$ denote the largest absolute value of the components of the vector $x$.

Proposition 1. Let $\alpha$ and $\beta$ be positive scalars, let $A=\operatorname{diag}\left(a_{1}, a_{2}, a_{3}\right)$ be a diagonal positive-definite matrix with distinct diagonal entries. Let $K_{\mathrm{p}}$ and $K_{\mathrm{v}}(\omega)$ be given by

$$
K_{\mathrm{p}}=\frac{\alpha}{\operatorname{tr} A}
$$

and

$$
K_{\mathrm{v}}(\omega)=\beta\left[\begin{array}{ccc}
f\left(\omega_{1}\right) & 0 & 0 \\
0 & f\left(\omega_{2}\right) & 0 \\
0 & 0 & f\left(\omega_{3}\right)
\end{array}\right],
$$

where $f: \mathbb{R} \rightarrow \mathbb{R}$ is a given continuous function and for all $\omega \in \mathbb{R}^{3}$,

$$
\left|f\left(\omega_{i}\right) \omega_{i}\right| \leq 1, i=1,2,3
$$

Then, for all $t \geq 0$, the control torque given by (12) satisfies:

$$
\|u(t)\|_{\infty} \leq \frac{\alpha+\beta}{\sigma_{\min }(B)} .
$$

Proof. Note that

$$
\begin{aligned}
\|u(t)\|_{\infty} & \leq \sigma_{\max }\left(B^{-1}\right)\left\|K_{\mathrm{p}} S(t)+K_{\mathrm{v}} \omega(t)\right\|_{\infty} \\
& \leq \frac{1}{\sigma_{\min }(B)}\left(K_{\mathrm{p}}\|S(t)\|_{\infty}+\left\|K_{\mathrm{v}} \omega(t)\right\|_{\infty}\right) \\
& \leq \frac{1}{\sigma_{\min }(B)}\left(\frac{\alpha}{\operatorname{tr} A} \sum_{i=1}^{3}\left\|a_{i}\left[\tilde{R}^{\mathrm{T}}(t) e_{i}\right] \times e_{i}\right\|_{\infty}+\left\|\beta\left[\begin{array}{ccc}
f\left(\omega_{1}\right) & 0 & 0 \\
0 & f\left(\omega_{2}\right) & 0 \\
0 & 0 & f\left(\omega_{3}\right)
\end{array}\right]\left[\begin{array}{l}
\omega_{1} \\
\omega_{2} \\
\omega_{3}
\end{array}\right]\right\|_{\infty}\right) \\
& \leq \frac{1}{\sigma_{\min }(B)}\left(\frac{\alpha}{\operatorname{tr} A} \sum_{i=1}^{3} a_{i}+\beta\left\|\left[\begin{array}{l}
f\left(\omega_{1}\right) \omega_{1} \\
f\left(\omega_{2}\right) \omega_{2} \\
f\left(\omega_{3}\right) \omega_{3}
\end{array}\right]\right\|_{\infty}\right) \\
& =\frac{1}{\sigma_{\min }(B)}\left(\alpha+\beta \max _{i=1,2,3}\left(\left|f\left(\omega_{i}\right) \omega_{i}\right|\right)\right) \\
& \leq \frac{\alpha+\beta}{\sigma_{\min }(B)} .
\end{aligned}
$$

In the presence of saturation, we require that, for all $t \geq 0$,

$$
\|u(t)\|_{\infty} \leq u_{\max }
$$

where $u_{\max }$ denotes the torque control bound for each component $u_{i}(t)$ of $u(t)$. To enforce (19), we thus require that

$$
\frac{\alpha+\beta}{\sigma_{\min }(B)} \leq u_{\max }
$$

The bound (18) applies to each component of the control vector $u$. The following result provides a tighter bound on the components of $u$. 
Proposition 2. For $i=1,2,3$, let the $i$ th component of the control torque $u_{i}$ be given by

$$
u_{i}(t)=-b_{i}^{\prime}\left(K_{\mathrm{p}} S(t)+K_{\mathrm{v}} \omega(t)\right) .
$$

where $b_{i}^{\prime}$ is the $i$ th row of the matrix $B^{-1}$. Then, for all $t \geq 0$, the torque given by (21) satisfies

$$
\left|u_{i}(t)\right| \leq\left\|b_{i}^{\prime}\right\|(\alpha+\sqrt{3} \beta), \text { for } \mathrm{i}=1,2,3,
$$

where $\left\|b_{i}^{\prime}\right\|$ is the 2-norm of vector $b_{i}^{\prime}$.

Proof. Note that

$$
\begin{aligned}
& \left|u_{i}(t)\right| \leq\left\|b_{i}^{\prime}\right\|\left\|K_{\mathrm{p}} S(t)+K_{\mathrm{v}} \omega(t)\right\| \\
& \leq\left\|b_{i}^{\prime}\right\|\left(K_{\mathrm{p}}\|S(t)\|+\left\|K_{\mathrm{v}} \omega(t)\right\|\right) \\
& =\left\|b_{i}^{\prime}\right\|\left(\frac{\alpha}{\operatorname{tr} A}\left\|\sum_{i=1}^{3} a_{i}\left[\tilde{R}^{\mathrm{T}}(t) e_{i}\right] \times e_{i}\right\|+\left\|\beta\left[\begin{array}{ccc}
f\left(\omega_{1}\right) & 0 & 0 \\
0 & f\left(\omega_{2}\right) & 0 \\
0 & 0 & f\left(\omega_{3}\right)
\end{array}\right]\left[\begin{array}{l}
\omega_{1} \\
\omega_{2} \\
\omega_{3}
\end{array}\right]\right\|\right) \\
& \leq\left\|b_{i}^{\prime}\right\|\left(\frac{\alpha}{\operatorname{tr} A} \sum_{i=1}^{3} a_{i}+\beta\left\|\left[\begin{array}{l}
f\left(\omega_{1}\right) \omega_{1} \\
f\left(\omega_{2}\right) \omega_{2} \\
f\left(\omega_{3}\right) \omega_{3}
\end{array}\right]\right\|\right) \\
& \leq\left\|b_{i}^{\prime}\right\|(\alpha+\sqrt{3} \beta) .
\end{aligned}
$$

Thus, in the presence of saturation,we require that

$$
\left\|b_{i}^{\prime}\right\|(\alpha+\sqrt{3} \beta) \leq u_{\max , i}, \text { for } i=1,2,3 .
$$

where $u_{\max , i}$ is the $i$ th component of the saturation limit vector $u_{\max }$.

Propositions 1 and 2 and Theorem 1 show that the spacecraft can be brought to rest with a desired attitude by an arbitrarily small torque using an inertia-free control law. However, fixing $\alpha$ and $\beta$ to satisfy the torque magnitude limits may result in unnecessarily slow response for maneuvers that do not violate the torque constraints.

We wish to choose $K_{\mathrm{v}}(\omega)$ and $K_{\mathrm{p}}$ so that the torque specified by (12) satisfies the bound (18). In [1], $f\left(\omega_{i}\right)$ is chosen as

$$
f\left(\omega_{i}\right)=\frac{1}{1+\left|\omega_{i}\right|}, i=1,2,3
$$

In the present paper, we consider the function

$$
f\left(\omega_{i}\right)= \begin{cases}\frac{1}{\bar{\omega}}, & 0 \leq\left|\omega_{i}\right| \leq \bar{\omega}, \\ \frac{1}{\left|\omega_{i}\right|}, & \left|\omega_{i}\right|>\bar{\omega}\end{cases}
$$

for $i=1,2,3$, where $\bar{\omega}$ is a positive scalar. We note that both (24) and (25) satisfy Proposition 1 . The advantage of (25) is that it provides a higher gain when the angular rates are small.

\section{Motion-to-Rest Example}

The following examples illustrate the result in Proposition 1 and expand it to better deal with saturation constraints. Proposition 2 can also be used to implement axis specific limits. 
We consider the objective of bringing the spacecraft from an arbitrary initial attitude and angular velocity to rest with a specified attitude. Hence, the desired attitude $R_{\mathrm{d}}$ is constant and the desired angular rate is $\omega_{\mathrm{d}}=0$ also we assume that the disturbance $z_{d}$ is zero.

Let the inertia matrix $J$ be given by

$$
J=\left[\begin{array}{ccc}
5 & -0.1 & -0.5 \\
-0.1 & 2 & 1 \\
-0.5 & 1 & 3.5
\end{array}\right] \mathrm{kg}-\mathrm{m}^{2},
$$

and $B=I$. We wish to bring the spacecraft from the initial attitude $R_{0}=I$ and initial angular velocity

$$
\omega(0)=\left[\begin{array}{lll}
1 & -1 & 0.5
\end{array}\right]^{\mathrm{T}} \mathrm{rad} / \mathrm{sec}
$$

to rest at the desired final orientation $R_{\mathrm{d}}=\operatorname{diag}(1,-1,-1)$. We choose $\bar{\omega}=0.2 \mathrm{rad} / \mathrm{sec}, A=\operatorname{diag}(1,2,3)$, and $\alpha=\beta=\frac{1}{2} u_{\max }$, where $u_{\max }=1-\mathrm{N}-\mathrm{m}$. The eigenaxis attitude errors, angular velocity, torque inputs, the value of Lyapunov function candidate, and its derivative are shown in Figure 1.

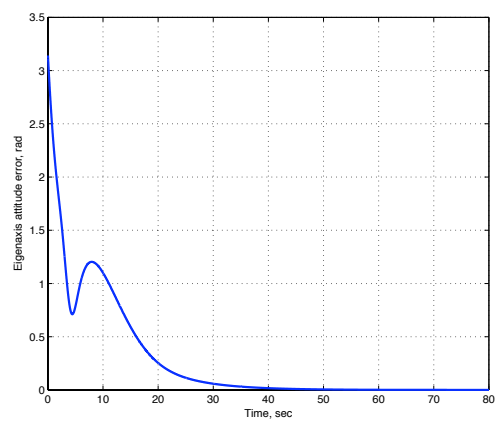

(a) Eigenaxis attitude error

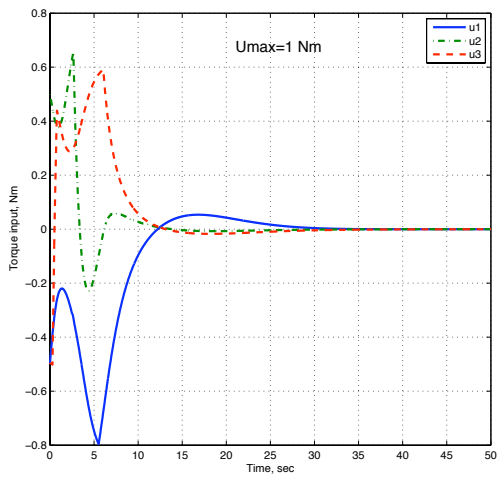

(c) Torque input

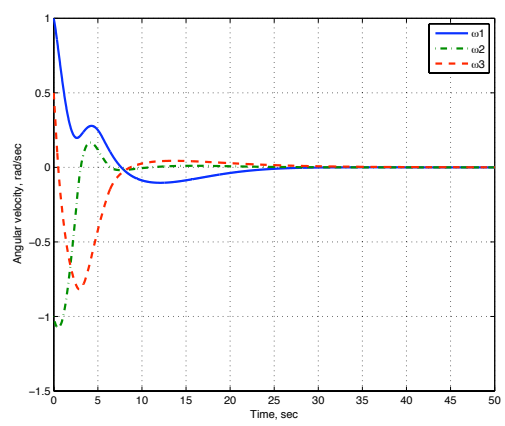

(b) Angular velocity
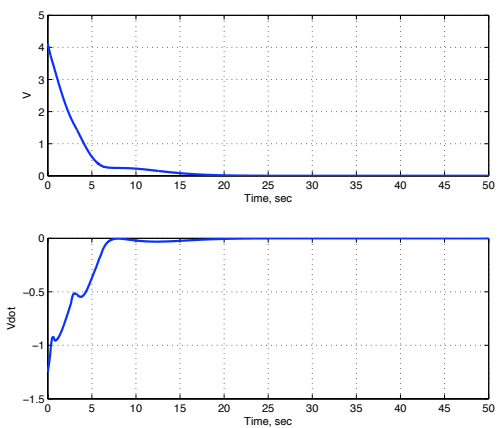

(d) $V$ and $\dot{V}$

Figure 1: Performance for the M2R algorithm with a saturation limit of 1-N-m.

Next, we decrease the saturation limit to $u_{\max }=0.1 \mathrm{~N}-\mathrm{m}$. Figure 2 shows the resulting performance. As expected, performance degrades relative to the case with $u_{\max }=1-\mathrm{N}-\mathrm{m}$ as indicated by the oscillations in the eigenaxis attitude error. These oscillations are due to the spacecraft spinning past $2 \pi$ while the controller reduces the spin rate to zero. Thus, convergence to the desired orientation is slower. 


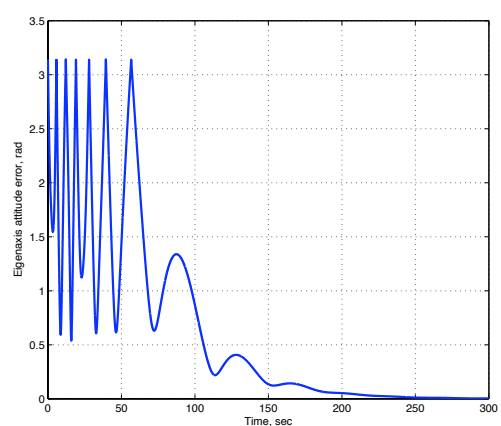

(a) Eigenaxis attitude error

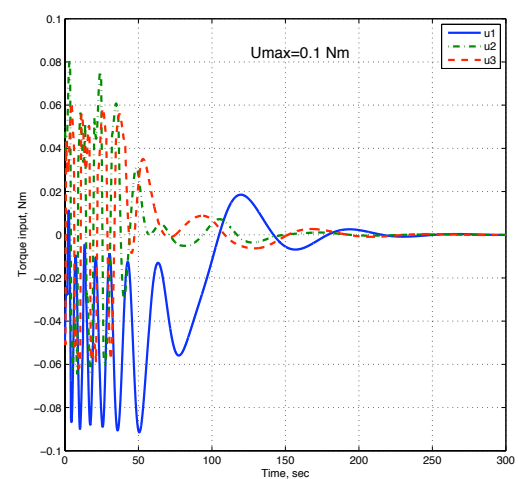

(c) Torque input

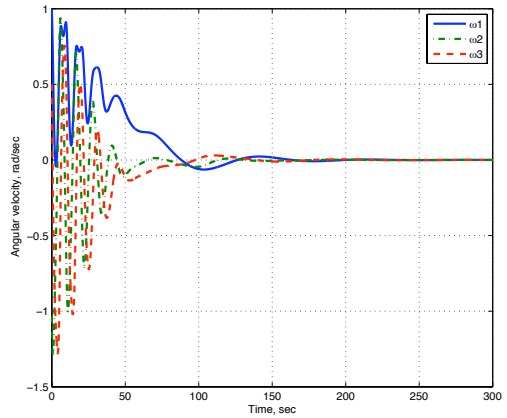

(b) Angular velocity
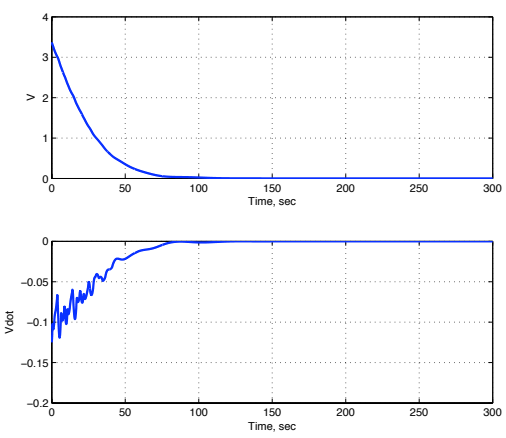

(d) $V$ and $\dot{V}$

Figure 2: Performance for the M2R control law with a saturation limit of 0.1-N-m.

Next, we consider the case $\alpha \neq \beta$. For convenience, we introduce a scalar $0 \leq \delta \leq 1$ so that

$$
\alpha=\delta u_{\max }
$$

and

$$
\beta=(1-\delta) u_{\max }
$$

To evaluate the effect of $\delta$, we introduce the performance metric

$$
k_{0} \triangleq \min \{k>200: \max [e(k-i)<0.03 \mathrm{rad}], i=1,2, \ldots, 200\},
$$

where $k$ is the time step and $e(t)$ is the eigenaxis attitude error. The metric $k_{0}$ can be interpreted as the minimal time such that the average attitude error during the most recent 200 time steps is less than 0.03 rad. For this maneuver and a saturation limit of $u_{\max }=1-\mathrm{N}-\mathrm{m}$, Figure 3 shows that the best performance is obtained for $\delta=0.4$. As these and other simulation results demonstrate, the choice of $\delta$ away from the endpoints of the interval $[0,1]$ results in faster maneuvers.

\subsection{Performance under a constant disturbance}

Next, we consider the effect of an unmodeled constant torque disturbance $z_{d}$ so that (1) is replaced by

$$
J \dot{\omega}=(J \omega) \times \omega+B u+z_{d}
$$




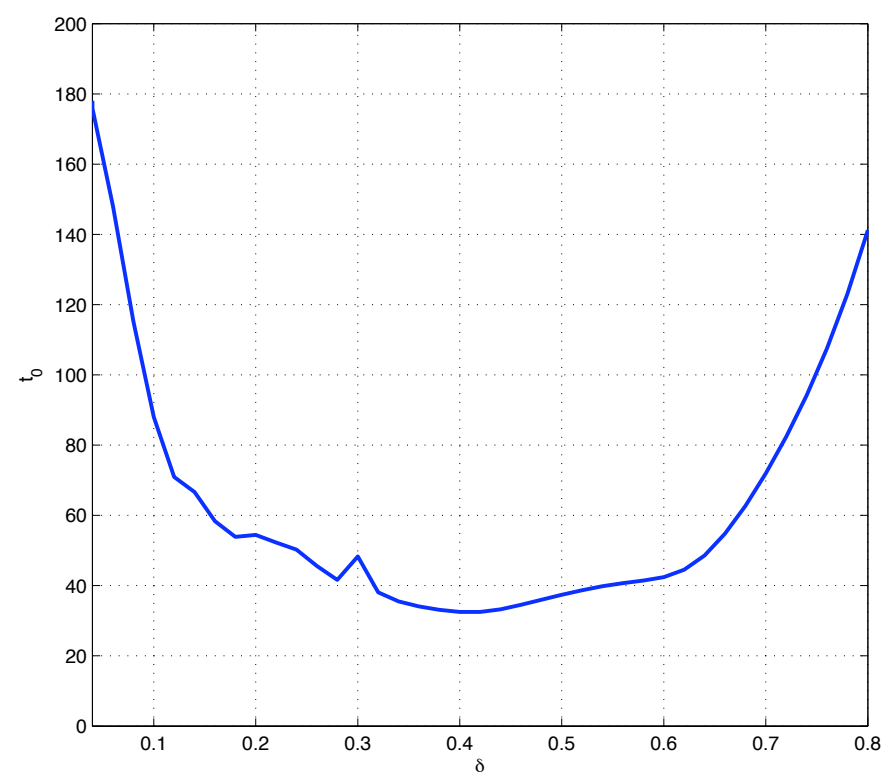

Figure 3: Performance metric $k_{0}$ as a function of $\delta$ for the M2R control law with a 1-N-m saturation limit.

Let $u_{\max }=1-\mathrm{N}-\mathrm{m}$ and $z_{d}=\left[\begin{array}{ccc}0.1 & 0.1 & 0.1\end{array}\right]^{\mathrm{T}} \mathrm{N}-\mathrm{m}$. Figure 4 shows the performance of the M2R control law in the presence of this disturbance. Note that the eigenaxis attitude error does not reach zero but approaches a steady state error. This error is due to the lack of integral action in the controller. In order to reduce the steady state error we increase $K_{\mathrm{p}}$ by choosing $A=\operatorname{diag}(0.1,0.2,0.3)$. Figure 5 shows that increasing $K_{\mathrm{p}}$ improves the attitude control performance.

Next, we increase the disturbance torque to $z_{d}=\left[\begin{array}{lll}0.2 & 0.2 & 0.2\end{array}\right]^{\mathrm{T}} \mathrm{N}-\mathrm{m}$ and set $A=\operatorname{diag}(1,2,3)$. In this case, the spacecraft aproaches a spin motion as shown by Figure 6 . which is undesired since we comanded an angular rate of zero. As shown in Figure 7, increasing $K_{\mathrm{p}}$ by choosing $A=\operatorname{diag}(0.1,0.2,0.3)$ yields improved performance.

\section{Motion-to-Spin (M2S) Control Law}

We define the notation

$$
J \omega=L(\omega) \gamma
$$

where $\gamma \in \mathbb{R}^{6}$ is defined by

$$
\gamma \triangleq\left[\begin{array}{llllll}
J_{11} & J_{22} & J_{33} & J_{23} & J_{13} & J_{12}
\end{array}\right]^{\mathrm{T}}
$$

and

$$
L(\omega) \triangleq\left[\begin{array}{cccccc}
\omega_{1} & 0 & 0 & 0 & \omega_{3} & \omega_{2} \\
0 & \omega_{2} & 0 & \omega_{3} & 0 & \omega_{1} \\
0 & 0 & \omega_{3} & \omega_{2} & \omega_{1} & 0
\end{array}\right]
$$




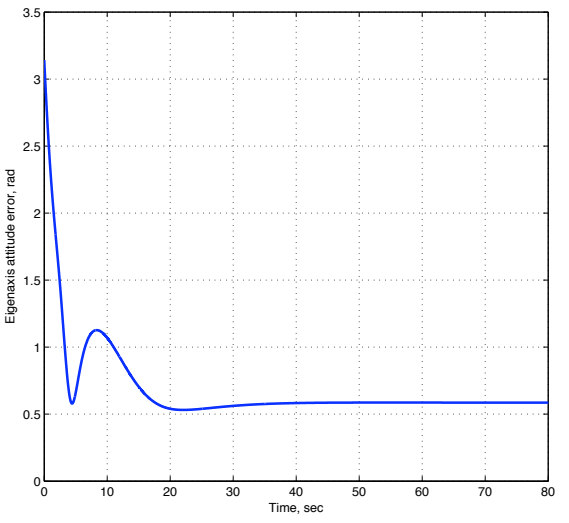

(a) Eigenaxis attitude error

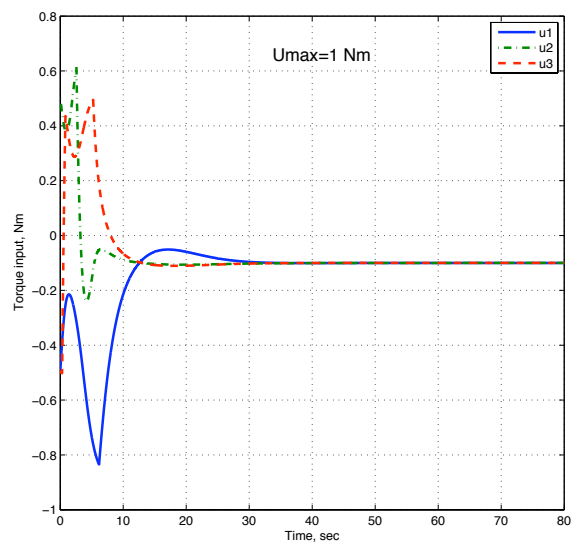

(c) Torque input

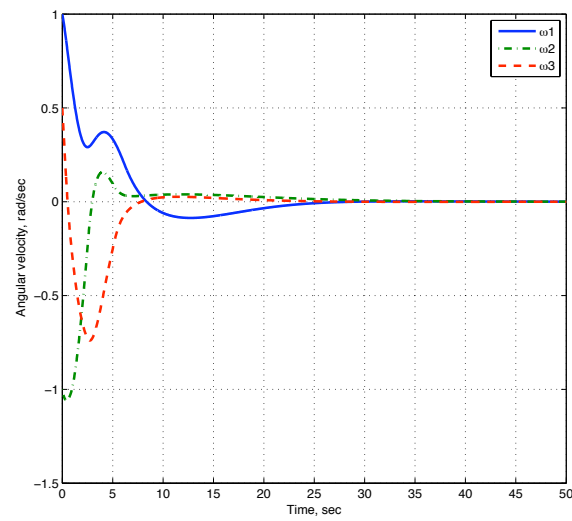

(b) Angular velocity
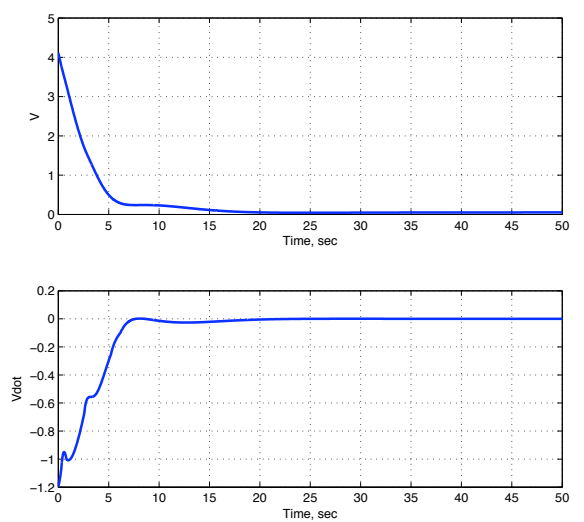

(d) $V$ and $\dot{V}$

Figure 4: M2R response in the presence of a constant disturbance $z_{d}=\left[\begin{array}{lll}0.1 & 0.1 & 0.1\end{array}\right]^{\mathrm{T}} \mathrm{N}-\mathrm{m}$ with $A=$ $\operatorname{diag}(1,2,3)$.

With this notation, (9) can be rewritten as

$$
J \dot{\tilde{\omega}}=\left[L\left(\tilde{\omega}+\tilde{R}^{\mathrm{T}} \omega_{\mathrm{d}}\right) \gamma\right]^{\times}\left(\tilde{\omega}+\tilde{R}^{\mathrm{T}} \omega_{\mathrm{d}}\right)+L\left(\tilde{\omega} \times \tilde{R}^{\mathrm{T}} \omega_{\mathrm{d}}-\tilde{R}^{\mathrm{T}} \dot{\omega}_{\mathrm{d}}\right) \gamma+B u+z_{d} .
$$

Next, let $\hat{J} \in \mathbb{R}^{3 \times 3}$ denote an estimate of $J$, and define the inertia-estimation error

$$
\tilde{J} \triangleq J-\hat{J} .
$$

Letting $\hat{\gamma}, \tilde{\gamma} \in \mathbb{R}^{6}$ represent $\hat{J}, \tilde{J}$, respectively, it follows that

$$
\tilde{\gamma}=\gamma-\hat{\gamma}
$$

Likewise, let $\hat{z}_{d} \in \mathbb{R}^{3}$ denote an estimate of $z_{d}$, and define the disturbance-estimation error

$$
\tilde{z}_{d} \triangleq z_{d}-\hat{z}_{d}
$$

Assumption 3. Each component of $z_{d}$ is a linear combination of constant and harmonic signals, whose frequencies are known but whose amplitudes and phases are unknown. 


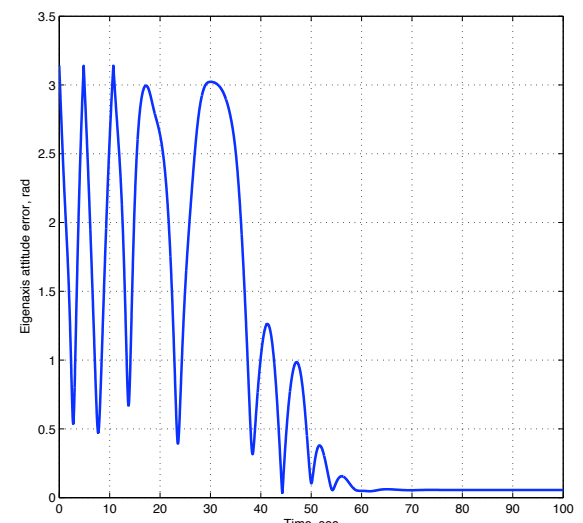

(a) Eigenaxis attitude error

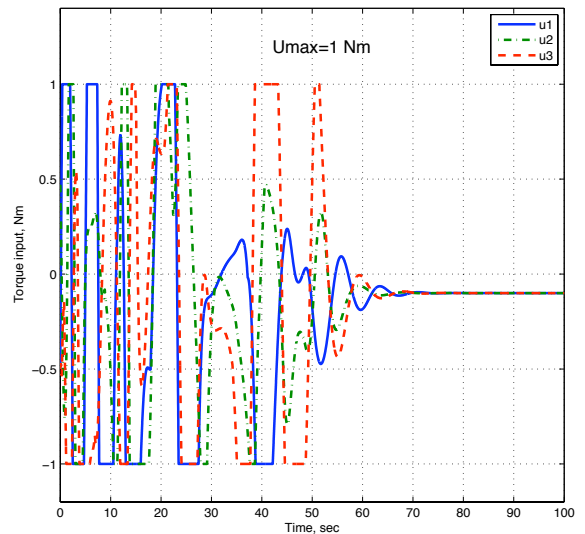

(c) Torque input

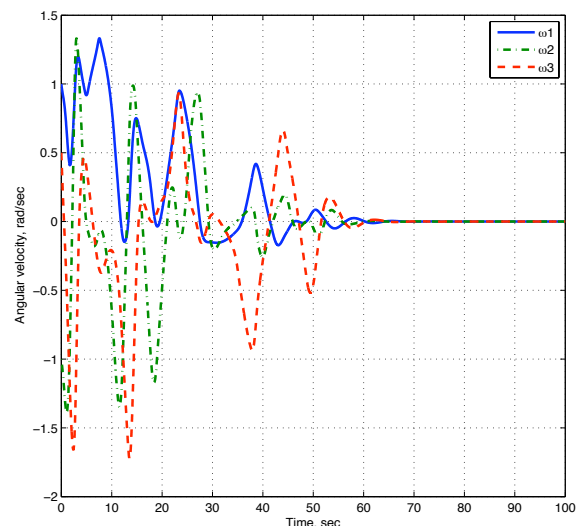

(b) Angular velocity
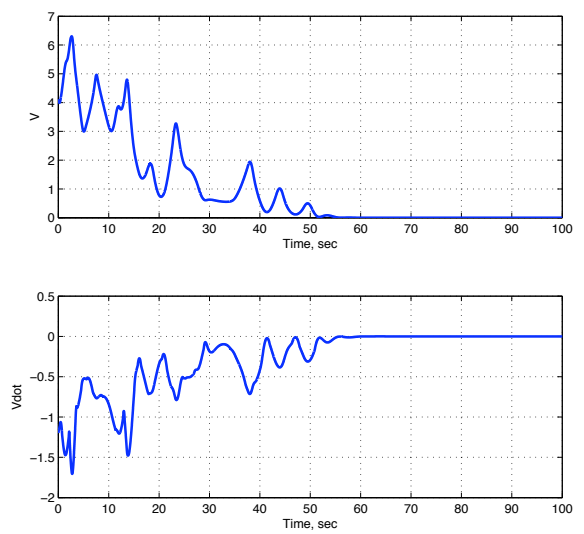

(d) $V$ and $\dot{V}$

Figure 5: Response of the M2R control law in the presence of the constant disturbance $z_{d}=\left[\begin{array}{lll}0.1 & 0.1 & 0.1\end{array}\right]^{\mathrm{T}}$ N-m with $A=\operatorname{diag}(0.1,0.2,0.3)$.

Assumption 3 implies that $z_{d}$ can be modeled as the output of an autonomous system of the form

$$
\begin{aligned}
\dot{d} & =A_{d} d, \\
z_{d} & =C_{d} d,
\end{aligned}
$$

where $A_{d} \in \mathbb{R}^{n_{d} \times n_{d}}$ and $C_{d} \in \mathbb{R}^{3 \times n_{d}}$ are known matrices and $A_{d}$ is a Lyapunov-stable matrix. In this model, $d(0)$ is unknown, which is equivalent to the assumption that the amplitude and phase of all harmonic components in the disturbance are unknown. The matrix $A_{d}$ is chosen to include eigenvalues of all frequency components that may be present in the disturbance signal, where the zero eigenvalue corresponds to constant disturbances. In effect, the controller provides infinite gain at the disturbance frequency, which results in asymptotic rejection of harmonic disturbance components. In particular, an integral controller provides infinite gain at DC in order to reject constant disturbances. In the case of orbit-dependent disturbances, the frequencies can be estimated from the orbital parameters. Likewise, in the case of disturbances originating from on-board devices, the spectral content of the disturbances may be known. In other cases, it may be possible to estimate the spectrum of the disturbances through signal processing. Assumption 3 implies that $A_{d}$ can be chosen to be skew symmetric, which we do henceforth. Let $\hat{d} \in \mathbb{R}^{n_{d}}$ denote an estimate of $d$, and define the disturbance-state estimation error

$$
\tilde{d} \triangleq d-\hat{d}
$$




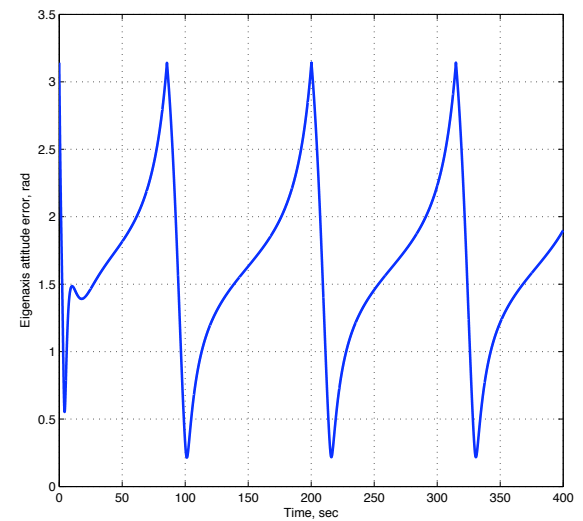

(a) Eigenaxis attitude error

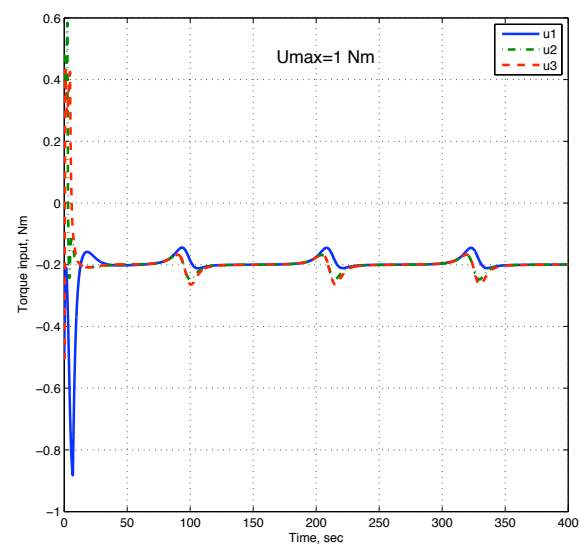

(c) Torque input

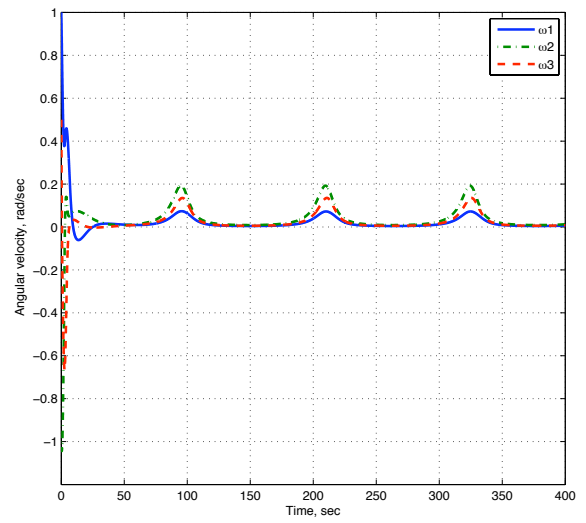

(b) Angular velocity
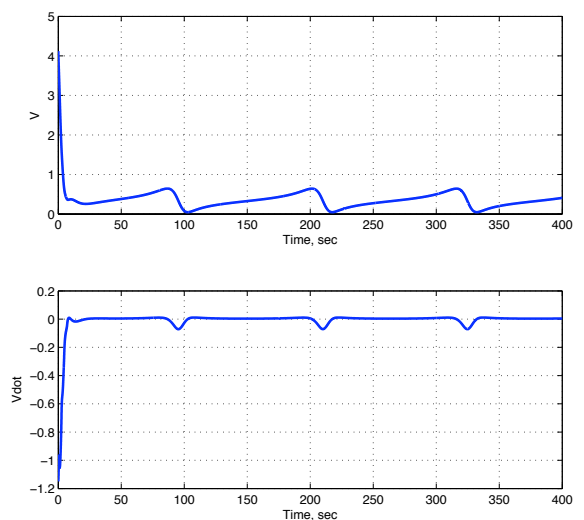

(d) $V$ and $\dot{V}$

Figure 6: Response of the M2R control law in the presence of the constant disturbance $z_{d}=\left[\begin{array}{lll}0.2 & 0.2 & 0.2\end{array}\right]^{\mathrm{T}}$ N-m with $A=\operatorname{diag}(1,2,3)$.

For $i=1,2,3$, let $e_{i}$ denote the $i$ th column of the $3 \times 3$ identity matrix. The following results are proven in [1].

Theorem 1. Let $K_{\mathrm{p}}$ be a positive scalar, let $K_{1} \in \mathbb{R}^{3 \times 3}$, let $Q \in \mathbb{R}^{6 \times 6}$ and $D \in \mathbb{R}^{n_{d} \times n_{d}}$ be positive definite, let $A=\operatorname{diag}\left(a_{1}, a_{2}, a_{3}\right)$ be a diagonal positive-definite matrix, and define

$$
S \triangleq \sum_{i=1}^{3} a_{i}\left(\tilde{R}^{\mathrm{T}} e_{i}\right) \times e_{i} .
$$

Then the Lyapunov function candidate

$$
V(\tilde{\omega}, \tilde{R}, \tilde{\gamma}, \tilde{d}) \triangleq \frac{1}{2}\left(\tilde{\omega}+K_{1} S\right)^{\mathrm{T}} J\left(\tilde{\omega}+K_{1} S\right)+K_{\mathrm{p}} \operatorname{tr}(A-A \tilde{R})+\frac{1}{2} \tilde{\gamma}^{\mathrm{T}} Q \tilde{\gamma}+\frac{1}{2} \tilde{d}^{\mathrm{T}} D \tilde{d}
$$

is positive definite, that is, $V$ is nonnegative, and $V=0$ if and only if $\tilde{\omega}=0, \tilde{R}=I, \tilde{\gamma}=0$, and $\tilde{d}=0$.

Theorem 2. Let $K_{\mathrm{p}}$ be a positive scalar, let $K_{\mathrm{v}} \in \mathbb{R}^{3 \times 3}, K_{1} \in \mathbb{R}^{3 \times 3}, Q \in \mathbb{R}^{6 \times 6}$, and $D \in \mathbb{R}^{n_{d} \times n_{d}}$ be positive definite, assume that $A_{d}^{\mathrm{T}} D+D A_{d}$ is negative semidefinite, let $A=\operatorname{diag}\left(a_{1}, a_{2}, a_{3}\right)$ be a diagonal 


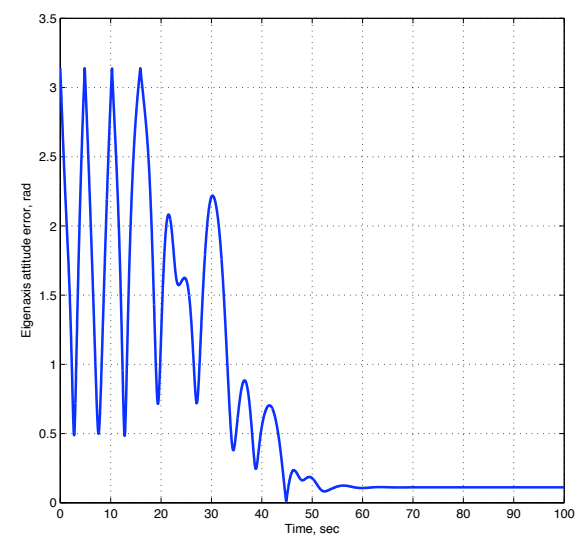

(a) Eigenaxis attitude error

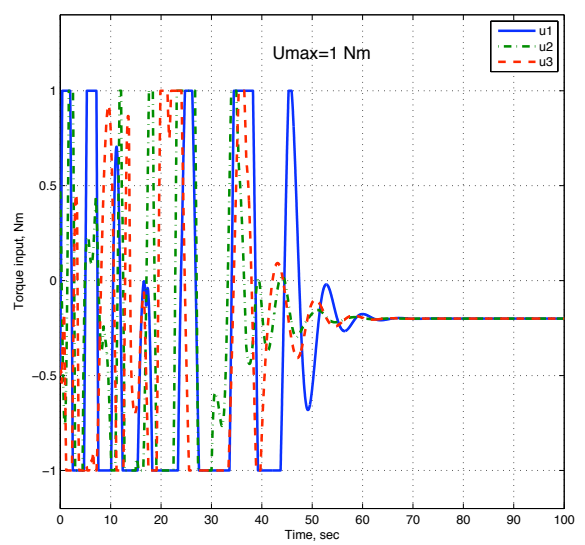

(c) Torque input

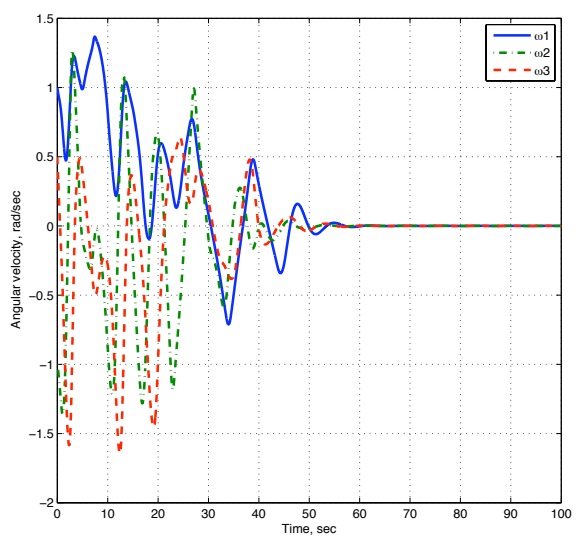

(b) Angular velocity
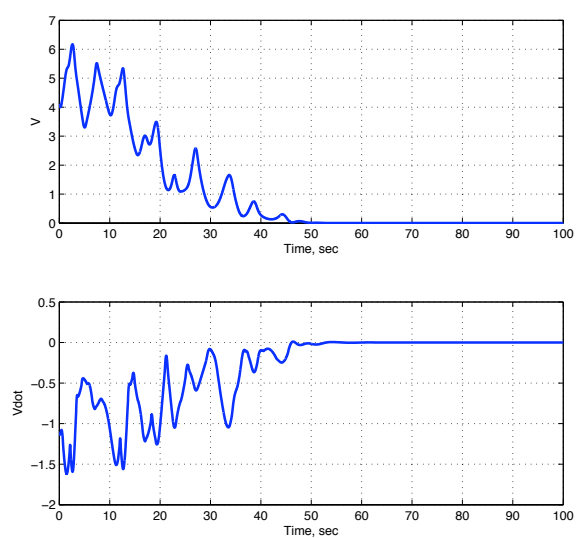

(d) $V$ and $\dot{V}$

Figure 7: Response of the M2R control law in the presence of the constant disturbance $z_{d}=\left[\begin{array}{lll}0.2 & 0.2 & 0.2\end{array}\right]^{\mathrm{T}}$ $\mathrm{N}-\mathrm{m}$ and $A=\operatorname{diag}(0.1,0.2,0.3)$. 
positive-definite matrix, define $S$ and $V$ as in Theorem 1 , and let $\hat{\gamma}$ and $\hat{d}$ satisfy

$$
\dot{\hat{\gamma}}=Q^{-1}\left[L^{\mathrm{T}}(\omega) \omega^{\times}+L^{\mathrm{T}}\left(K_{1} \dot{S}+\tilde{\omega} \times \omega-\tilde{R}^{\mathrm{T}} \dot{\omega}_{\mathrm{d}}\right)\right]\left(\tilde{\omega}+K_{1} S\right),
$$

where

$$
\dot{S}=\sum_{i=1}^{3} a_{i}\left[\left(\tilde{R}^{\mathrm{T}} e_{i}\right) \times \tilde{\omega}\right] \times e_{i}
$$

and

$$
\begin{aligned}
\dot{\hat{d}} & =A_{d} \hat{d}+D^{-1} C_{d}^{\mathrm{T}}\left(\tilde{\omega}+K_{1} S\right), \\
\hat{z}_{d} & =C_{d} \hat{d} .
\end{aligned}
$$

Furthermore, let

$$
u=B^{-1}\left(v_{1}+v_{2}+v_{3}\right),
$$

where

$$
\begin{gathered}
v_{1} \triangleq-(\hat{J} \omega) \times \omega-\hat{J}\left(K_{1} \dot{S}+\tilde{\omega} \times \omega-\tilde{R}^{\mathrm{T}} \dot{\omega}_{\mathrm{d}}\right), \\
v_{2} \triangleq-\hat{z}_{d}
\end{gathered}
$$

and

$$
v_{3} \triangleq-K_{\mathrm{v}}\left(\tilde{\omega}+K_{1} S\right)-K_{\mathrm{p}} S
$$

Then,

$$
\dot{V}(\tilde{\omega}, \tilde{R}, \tilde{\gamma}, \tilde{d})=-\left(\tilde{\omega}+K_{1} S\right)^{\mathrm{T}} K_{\mathrm{v}}\left(\tilde{\omega}+K_{1} S\right)-K_{\mathrm{p}} S^{\mathrm{T}} K_{1} S+\frac{1}{2} \tilde{d}^{\mathrm{T}}\left(A_{d}^{\mathrm{T}} D+D A_{d}\right) \tilde{d}
$$

is negative semidefinite.

Note that the closed-loop spacecraft attitude dynamics with the controller given by Theorem 2 are given by

$$
J \dot{\tilde{\omega}}=[L(\omega) \tilde{\gamma}]^{\times} \omega+L\left(\tilde{\omega} \times \tilde{R}^{\mathrm{T}} \omega_{\mathrm{d}}-R^{\mathrm{T}} \dot{\omega}_{\mathrm{d}}\right) \tilde{\gamma}-L\left(K_{1} \dot{S}\right) \hat{\gamma}+\tilde{z}_{d}-K_{\mathrm{v}}\left(\tilde{\omega}+K_{1} S\right)-K_{\mathrm{p}} S .
$$

If $A_{d}$ is chosen to be skew symmetric, then choosing $D$ to be a multiple of the identity implies that $A_{d}^{\mathrm{T}} D+$ $D A_{d}=0$, and thus $\dot{V}$ is negative semidefinite.

\section{Motion-to-Spin Saturation Techniques}

\subsection{Torque Cutoff}

This algorithm cuts off torque commanded torque components whose magnitude exceed the saturation

limit. When a torque component exceeds the limit, the torque produced in that axis is set to the maximum torque allowed. Define the saturation function

$$
\operatorname{sat}\left(x, x_{\max }\right)=\left\{\begin{array}{l}
x, \quad|x| \leq x_{\max }, \\
\operatorname{sgn}(x) x_{\max }, \quad|x|>x_{\max },
\end{array}\right.
$$

where $x, x_{\max }$ are scalars and $x_{\max }>0$. Then if $u \in \mathbb{R}^{3}$ is the commanded torque from (41) the saturated control torque $u^{\prime}$ is

$$
u_{i}^{\prime}=\operatorname{sat}\left(u_{i}, u_{\max , i}\right), i=1,2,3 .
$$




\subsection{Torque Scaling}

The cutoff algorithm does not preserve the commanded torque vector's direction, which may negatively affect the control algorithm's performance. The scaling algorithm maximizes the magnitude of the torque vector while maintaining the original vector's direction.

Define a bounding region $B\left(x_{\max }\right) \in \mathbb{R}^{3}$, which represents the saturation limits for the vector $x$. We define the scaling function as

$$
\operatorname{scl}(x, B)= \begin{cases}x, & x \in B, \\ \alpha_{\max } x & x \notin B,\end{cases}
$$

where $\alpha_{\max }$ is defined as

$$
\alpha_{\max }=\max _{\alpha \in(0,1]}\{\alpha: \alpha x \in B\}
$$

Thus, the function scl scales the vector $x$ whenever it exceeds the saturation limits. The torque scaling algorithm is

$$
u^{\prime}=\operatorname{scl}\left(u, B\left(u_{\max }\right)\right)
$$

\subsection{Principal Axis Spin Example}

Let the inertia matrix $J$ be given by

$$
J=\left[\begin{array}{ccc}
1.4947 & 0 & 0 \\
0 & 3.7997 & 0 \\
0 & 0 & 5.2056
\end{array}\right] \mathrm{kg}-\mathrm{m}^{2} .
$$

Consider a desired spin maneuver with $B=I$, zero disturbances, and with the spacecraft initially tumbling with

$$
R(0)=\left[\begin{array}{ccc}
1 & 0 & 0 \\
0 & 0 & -1 \\
0 & 1 & 0
\end{array}\right]
$$

and

$$
\omega(0)=\frac{-0.1}{\sqrt{3}}\left[\begin{array}{lll}
1 & 1 & 1
\end{array}\right]^{\mathrm{T}} \mathrm{rad} / \mathrm{sec} .
$$

We choose $A=\operatorname{diag}(1,2,3), \alpha=\beta=1, K_{1}=D=I_{3}$, and $Q=I_{6}$. Furthermore, $\hat{J}(0)=I_{3}$, and the disturbance torques are not estimated by setting $C_{d}=0_{3 \times 3}$. With the above parameters, a spin about the intermediate principal axis is examined. The reference attitude is given by $R_{\mathrm{d}}(0)=I$ with desired constant angular velocity

$$
\omega_{\mathrm{d}}=0.1\left[\begin{array}{lll}
0 & 1 & 0
\end{array}\right]^{\mathrm{T}} \mathrm{rad} / \mathrm{sec} .
$$

Figure 8 compares the performance of the cutoff and scaling saturation techniques and shows the unsaturated performance for the maneuver. As saturation limits are decreased, maneuver speed is reduced and transients increase as command authority decreases. For principal axis spins, it is physically possible to perform the maneuver with arbitrarily small torques. However, the M2S controller has problems when the saturation limit is too low: the inertia estimate diverges and the controller cannot reach steady state. Figure 9 shows that both saturation methods fail for lower saturation limits, both the rate and attitude 


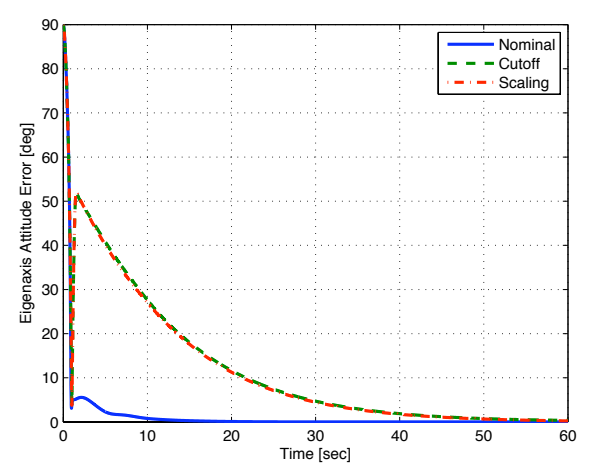

(a) Eigenaxis Attitude Error
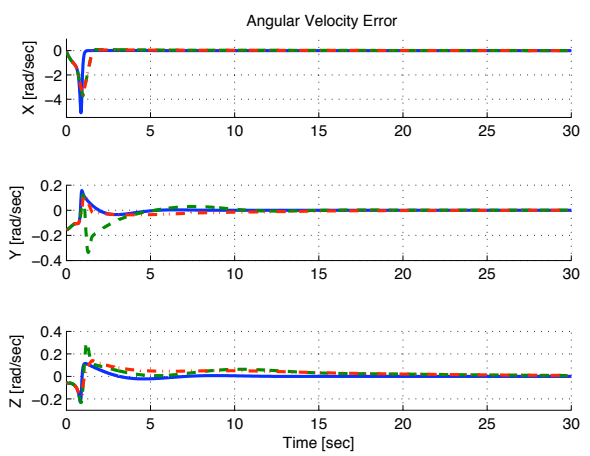

(b) Angular Rate Error

Figure 8: M2S about the intermediate principal axis with $\left\|\omega_{d}\right\|=0.1 \mathrm{rad} / \mathrm{sec}$ without disturbances. Performance is compared for the unsaturated case, the cutoff method and the scaling method with a 10-N-m saturation limit on all axes.

are unable to reach the desired values. Furthermore, Figure 8d shows the difference between the required torque from the controller and the torque produced by the saturation techniques, we can observe how for both methods the required torque diverges and is larger than the saturation limits. Finally, Figure 10 shows that the inertia estimates diverge and the Lyapunov function monotonically increases, indicating that the closed-loop system is unstable.

\section{Saturation Mitigation Techniques}

\subsection{Varying Adaptation Gain (VAG)}

We now address the divergence of the inertia estimates by modifying the adaptation rate. As the saturation level is decreased and the transients increase, the inertia estimate $\hat{\gamma}$ reacts and changes rapidly. The adaptation rate of change $\dot{\hat{\gamma}}$ can be decreased by modifying the value of $Q$ in (37). The motivation for decreasing the adaptation rate is derived from a related antiwindup handling technique of suspending the integrator update during actuator saturation. Define the adaptation rate matrix,

$$
Q=q I_{6},
$$

where $q$ is a positive scalar. Note that increasing the value $q$ decreases the adaptation rate. function

The following saturation law for the adaptation rate is proposed. Define the adaptation rate saturation

$$
\operatorname{qsat}\left(q, q_{\max }, u, u_{\max }\right)=\left\{\begin{array}{lc}
q, & u_{i} \in B\left(u_{\max }\right) \\
q_{\max }, & q \geq q_{\max }, \\
\left(1+K_{Q} \frac{\left\||u|-u_{\max }\right\|}{\left\|u_{\max }\right\|}\right) q, & q<q_{\max } \text { and } u_{i} \notin B\left(u_{\max }\right),
\end{array}\right.
$$

where $K_{Q}$ is a positive scalar. The adaptation parameter $q$ is not allowed to increase above a value $q_{\max }$; large $q$ causes numerical errors in the computation of the inverse term in (37). Furthermore, there is a point of diminishing returns as this limit is increased. This saturation technique decreases the adaptation rate 


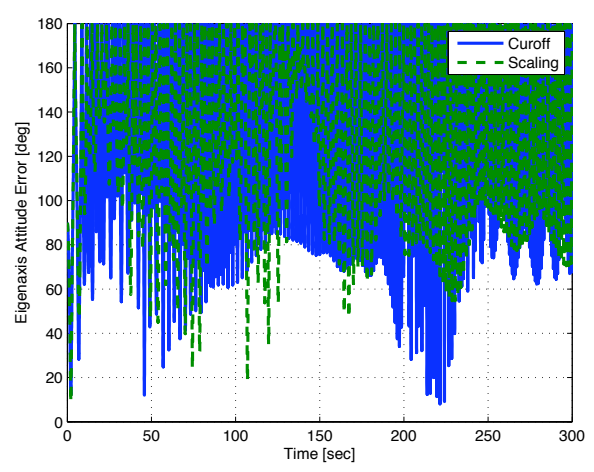

(a) Eigenaxis Attitude Error
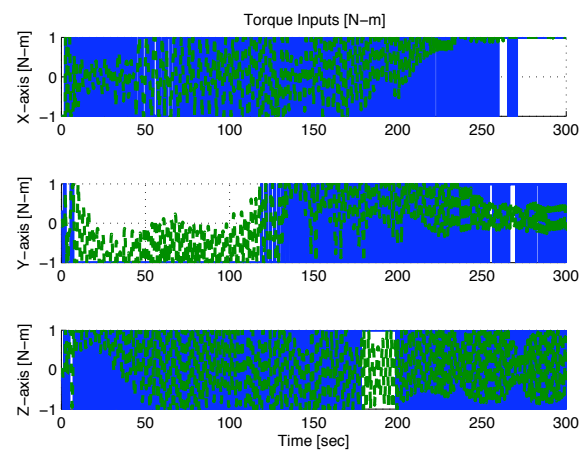

(c) Control Torque
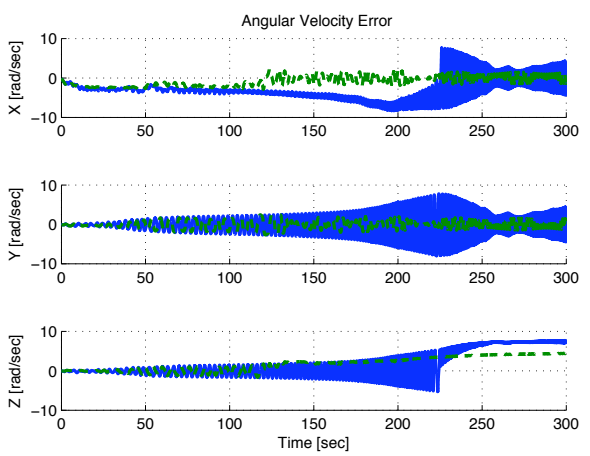

(b) Angular Rate Error
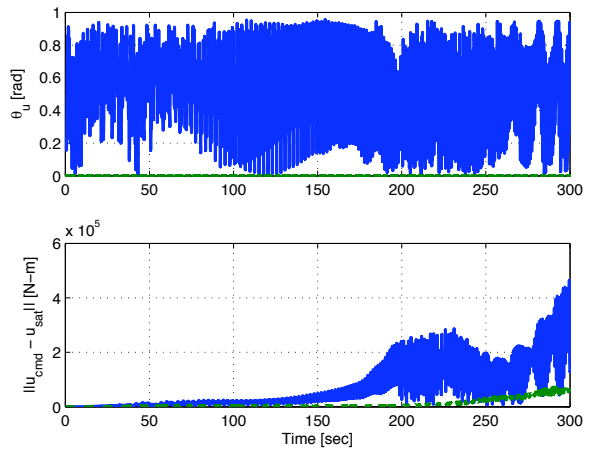

(d) Control Vector Angle and Magnitude Difference

Figure 9: M2S about the intermediate principal axis with $\left\|\omega_{d}\right\|=0.1 \mathrm{rad} / \mathrm{sec}$ without disturbances. The performance is compared for the cutoff method and scaling method with a $1-\mathrm{N}-\mathrm{m}$ saturation limit on all axes.

$\dot{\hat{\gamma}}$ whenever a saturation event occurs. The reduction is proportional to the excess torque required by the maneuver.

The VAG method combines the above saturation law with either the cutoff or the scaling method to manage and implement saturation limits. Thus there are two variants of the VAG algorithm: VAG-C(cutoff)

$$
\begin{aligned}
& u_{i}^{\prime}=\operatorname{sat}\left(u_{i}, u_{\max , i}\right), i=1,2,3, \\
& q_{k}=\operatorname{qsat}\left(q_{k-1}, q_{\max }, u, u_{\max }\right),
\end{aligned}
$$

and VAG-S(scaling)

$$
\begin{array}{lc}
u^{\prime}= & \operatorname{scl}\left(u, B\left(u_{\max }\right)\right), \\
q_{k}= & \operatorname{qsat}\left(q_{k-1}, q_{\max }, u, u_{\max }\right) .
\end{array}
$$

where $u$ is the commanded torque from (41) and $u^{\prime}$ is the saturated control torque, $k$ is the current timestep. The saturation law in (56) maintains $q$ positive. Thus $Q$ is positive definite, which keeps the Lyapunov function candidate in (36) positive definite.

\subsection{Principal Axis Spin Example}

The VAG algorithms are applied to the intermediate principal axis spin maneuver considered in Section 6.3. In the following examples the adaptation rate limit is set to $q_{\max }=10000$ and the saturation gain is 

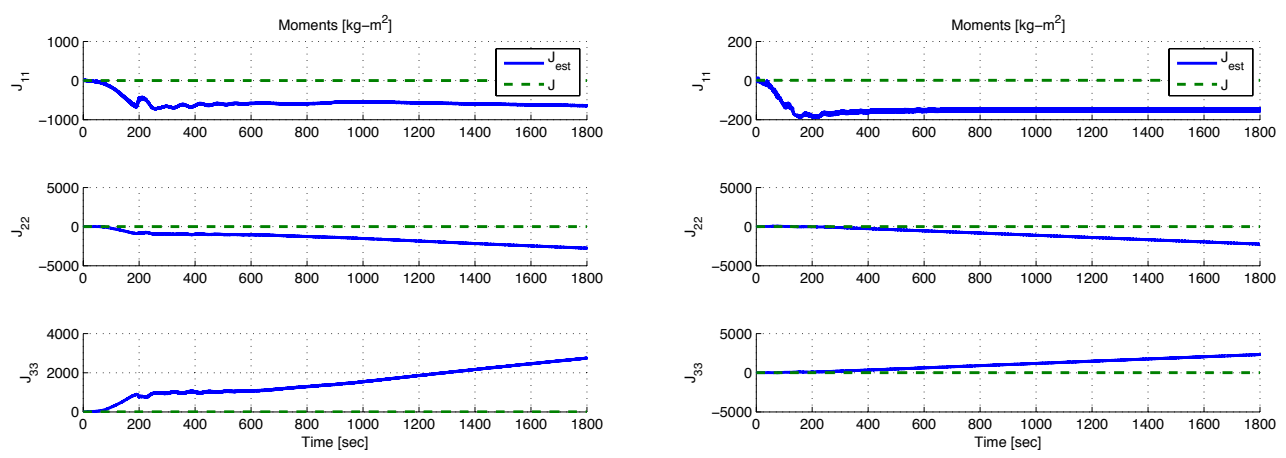

(a) Inertia Estimates for the Cutoff Method

(b) Inertia Estimates for the Scaling Method
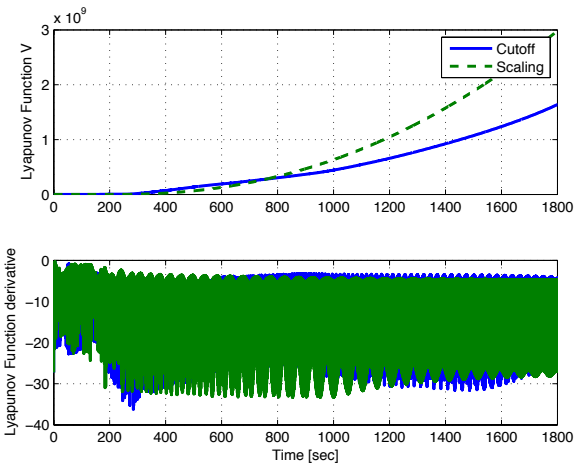

(c) Lyapunov Function and Derivative comparison

Figure 10: Moment of inertia estimates and Lyapunov functions from the Cutoff and Scaling methods for the intermediate principal axis spin maneuver with 1-N-m torque cutoff.

set to $K_{Q}=0.001$. Figure 11 shows the algorithm performance for saturation limits of $0.01 \mathrm{~N}-\mathrm{m}$.

\subsection{Arbitrary Axis Example}

Consider motion to spin maneuver for the desired spin rate

$$
\omega_{\mathrm{d}}=\frac{0.1}{\sqrt{3}}\left[\begin{array}{lll}
1 & 1 & 1
\end{array}\right]^{\mathrm{T}} \mathrm{rad} / \mathrm{sec} .
$$

The disturbances are zero and the controller parameters are chosen as in the previous examples. Figure 12 compares the performance of this algorithm with $u_{\max }=1-\mathrm{N}-\mathrm{m}$ against the nominal unsaturated spin maneuver. The body desired spin axis is the non principal axis $\left[\begin{array}{ccc}1 & 1 & 1\end{array}\right]^{\mathrm{T}}$.

The steady state torque requirement for this maneuver can be computed from Euler's equation

$$
\tau=J \dot{\omega}+\omega_{d} \times J \omega_{d}
$$

Setting $\dot{\omega}=0$ and using the desired rate in (59) yields

$$
\tau=\omega_{d} \times J \omega_{d}=\left[\begin{array}{lll}
0.0047 & -0.0124 & 0.0077
\end{array}\right]^{\mathrm{T}} \mathrm{rad} / \mathrm{sec} .
$$

Thus, the minimum feasible saturation limit is the infinity-norm of the steady state torque, $\|\tau\|_{\infty}=0.0124$ $\mathrm{N}-\mathrm{m}$. We thus examine the algorithm's behavior for saturation levels close to or below this value. Figure 13 


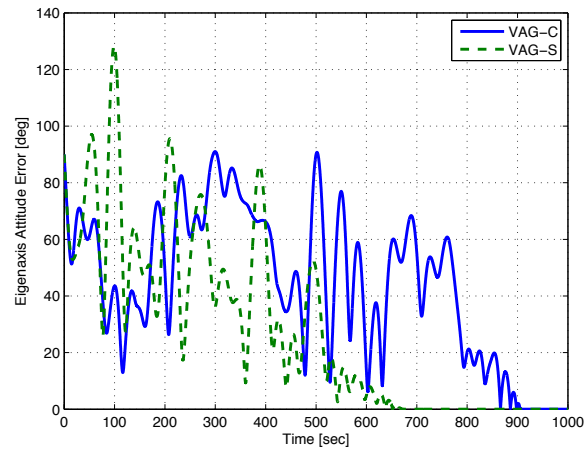

(a) Eigenaxis Attitude Error
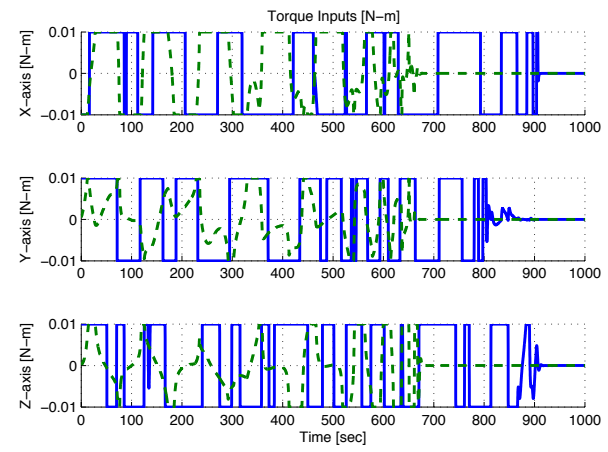

(c) Control Torque
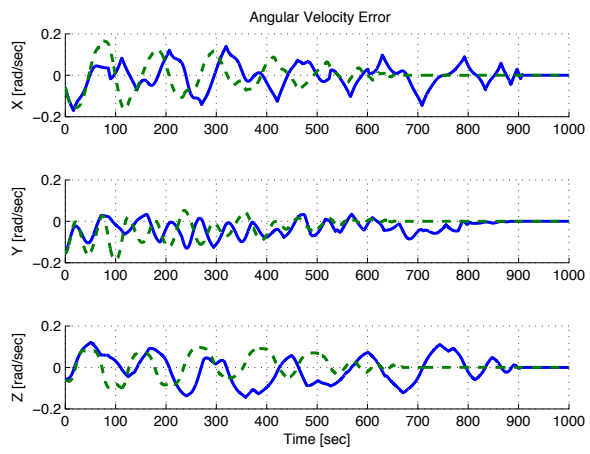

(b) Angular Rate Error
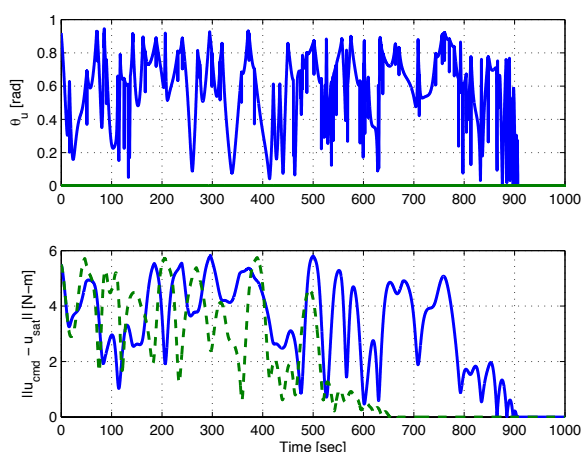

(d) Control Vector Comparison

Figure 11: M2S maneuver about the intermediate principal axis with $\left\|\omega_{d}\right\|=0.1 \mathrm{rad} / \mathrm{sec}$ without disturbances. Performance is compared for the VAG-C and the VAG-S methods for a $0.01 \mathrm{~N}-\mathrm{m}$ saturation limit on all axes.

examines the performance of the algorithm for $u_{\max }=0.02 \mathrm{~N}-\mathrm{m}$, which is close to the predicted minimum. Also, the infinity norm in Figure 13 (c) confirms that the steady state torque is close to the theoretical value $0.0124 \mathrm{~N}-\mathrm{m}$. Furthermore, when $u_{\max }$ decreases below this value the algorithm does not become unstable as does the the torque cutoff method. Instead, as shown in Figure 14, the closed-loop performance degrades gracefully converging to a spin about a different axis.

\subsection{Extremum Tracking}

The attitude error present in Figure 14 results from desired maneuvers which require more steady state torque than what is available. This occurs when the desired spin axis is not a principal axis. To eliminate the attitude error, the commanded spin rate can be reduced until the spacecraft reaches the desired spin axis.

Let the current angular rate be $\omega_{k}$ with direction $\hat{\omega}_{k}$. Define the current desired spin rate as $\bar{\omega}_{k}$ with direction $\hat{\omega}_{d, k}$ and the the maximum allowed angular rate direction error as $\tilde{\phi}_{\max }$. The current spin direction error is

$$
\tilde{\phi}_{k}=\operatorname{acos}\left(\hat{\omega}^{\mathrm{T}} \hat{\omega}_{d}\right)
$$




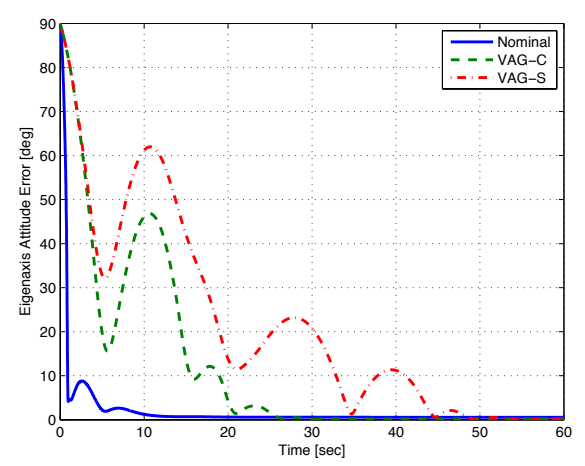

(a) Eigenaxis Attitude Error
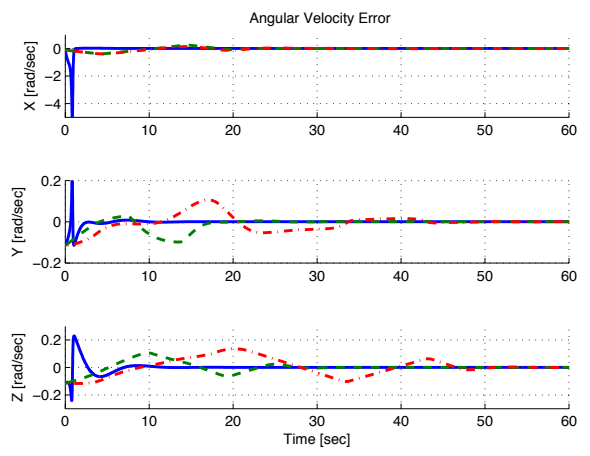

(b) Angular Rate Error

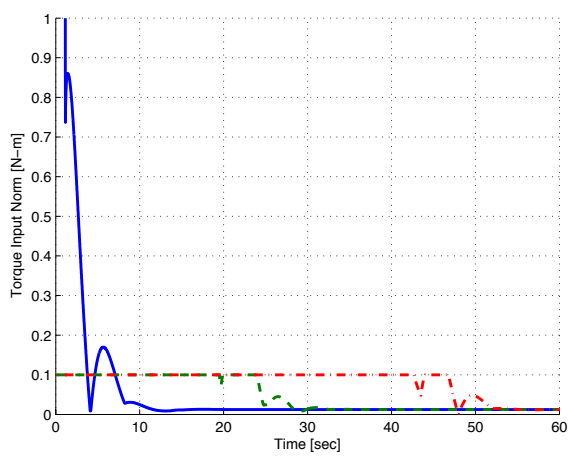

(c) Control Torque Infinity Norm

Figure 12: M2S maneuver about a non-principal body-fixed spin axis with $\left\|\omega_{d}\right\|=0.1 \mathrm{rad} / \mathrm{sec}$ without disturbances. Performance is compared for the unsaturated case and a $0.1 \mathrm{~N}-\mathrm{m}$ saturation limit imposed by the VAG-C and VAG-S algorithms.

To achieve the maneuver within the alloted torque constraints the a lower spin rate is commanded when we determine that the desired rate magnitude is not achievable. Define the maximum settling time $t_{\max }$, and the maximum allowable spin axis error $\tilde{\phi}_{\max }$. The time since the last rate command change is

$$
\Delta t=t-\bar{t}_{k},
$$

where $\bar{t}_{k}$ is the time of the last command change. Then the new commanded spin rate is

$$
\bar{\omega}_{k+1}=\left\{\begin{array}{l}
\bar{\omega}_{k}-\delta_{k+1}, \quad \tilde{\phi}_{k}>\tilde{\phi}_{\max } \text { AND } \Delta t>t_{\max } \\
\bar{\omega}_{k}+\delta_{k+1}, \quad \tilde{\phi}_{k} \leq \tilde{\phi}_{\max } \text { AND } \bar{\omega}_{k}<\bar{\omega}_{0} \text { AND } \Delta t>t_{\max } \\
\bar{\omega}_{k}, \quad \text { otherwise, }
\end{array}\right.
$$

where for $0<K_{\omega}<1$,

$$
\delta_{k+1}=K_{\omega} \delta_{k}
$$

and

$$
\alpha_{k+1}=\left\{\begin{array}{l}
1, \quad \tilde{\phi}_{k} \leq \tilde{\phi}_{\max } \\
-1, \quad \tilde{\phi}_{k}>\tilde{\phi}_{\max }
\end{array}\right.
$$

Using the maneuver from Section 7.3, the extremum tracking algorithm is combined with VAG-C to impose a $0.005 \mathrm{~N}-\mathrm{m}$ torque limit. The parameters for the extremum tracking algorithm in this example are 


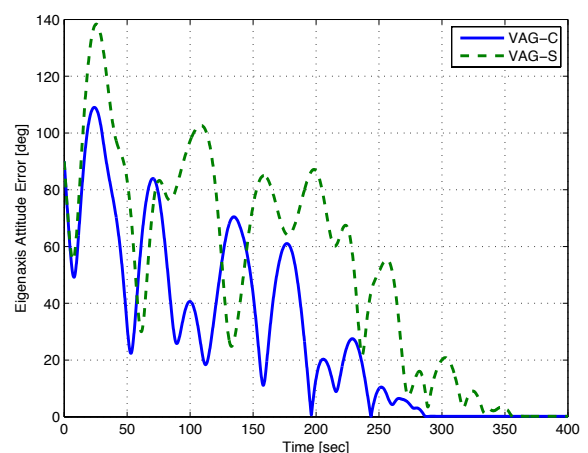

(a) Eigenaxis Attitude Error
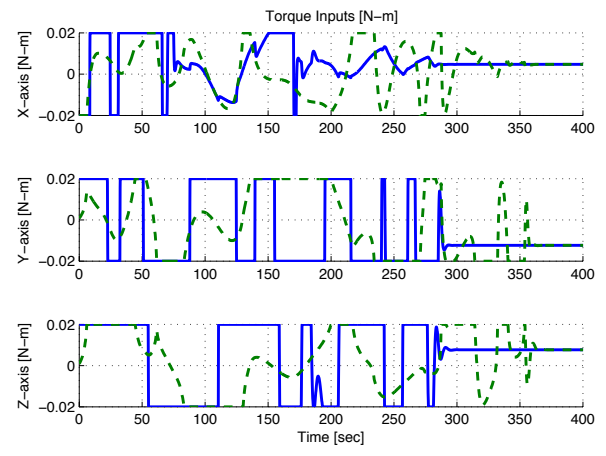

(c) Control Torque
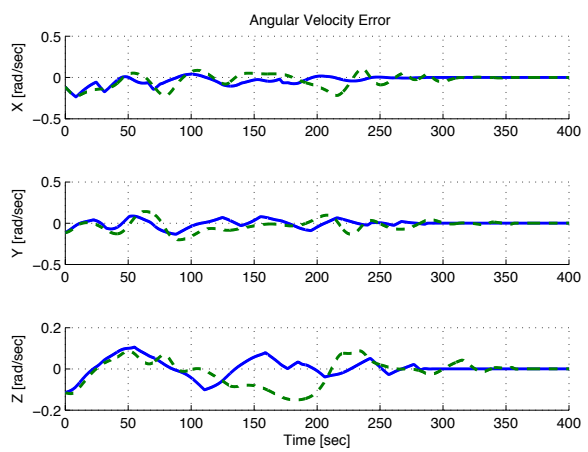

(b) Angular Rate Error
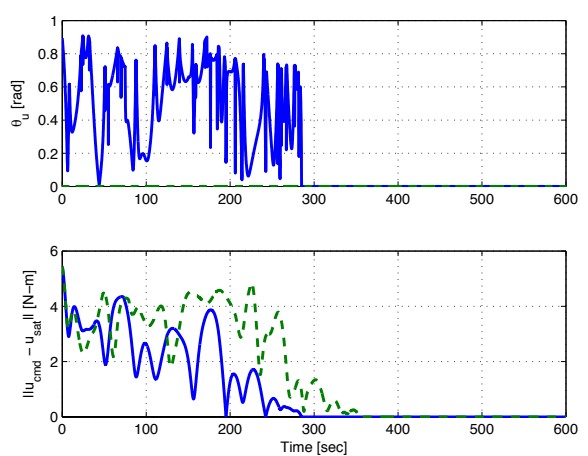

(d) Control Vector Comparison

Figure 13: M2S maneuver about a non-principal body-fixed spin axis with $\left\|\omega_{d}\right\|=0.1 \mathrm{rad} / \mathrm{sec}$ without disturbances. Performance is compared for the VAG-C and the VAG-S algorithms imposing a $0.02 \mathrm{~N}-\mathrm{m}$ saturation limit.

defined as

$$
\begin{array}{r}
t_{\max }=250 \mathrm{sec} \\
\tilde{\phi}_{\max }=10^{\circ} \\
K_{\omega}=0.5 .
\end{array}
$$

Figure 15 shows how the attitude error shown in Figure 14a can be eliminated by reducing the required spin rate.

\subsection{Performance under a constant disturbance}

For this example a constant disturbance torque is simulated, and the controller has an adaptive term to estimate the disturbance. The parameter $C_{D}=I_{3}$ and the constant disturbance is

$$
z_{d}=\frac{-0.1}{\sqrt{3}}\left[\begin{array}{lll}
1 & 1 & 1
\end{array}\right]^{\mathrm{T}} \mathrm{N}-\mathrm{m}
$$

Figure 16 compares the unsaturated performance of the M2S controller under a constant disturbance with the performance of the VAG-C algorithm imposing a 10-N-m saturation limit.

Imposing more stringent limits causes a similar issue as with the torque cutoff method. Figure 17 


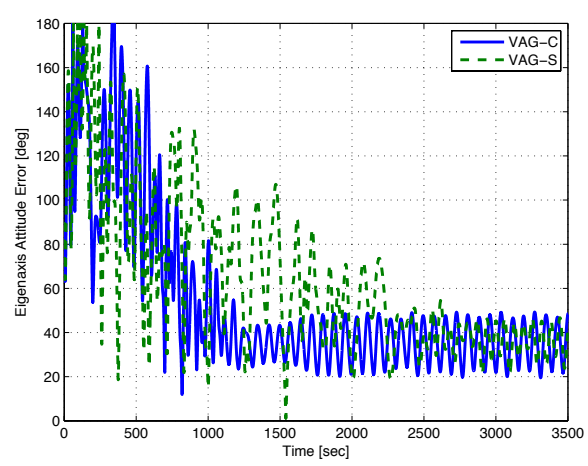

(a) Eigenaxis Attitude Error
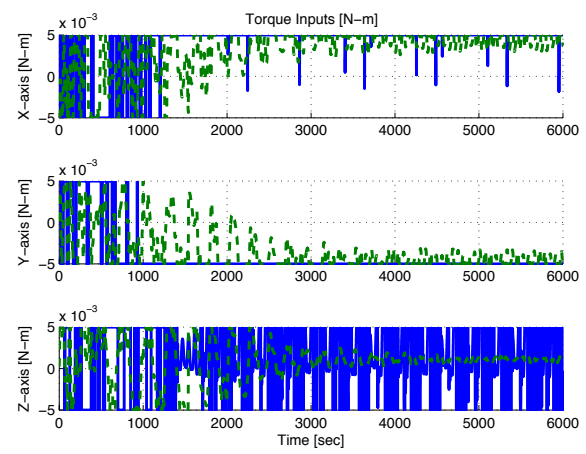

(c) Control Torque
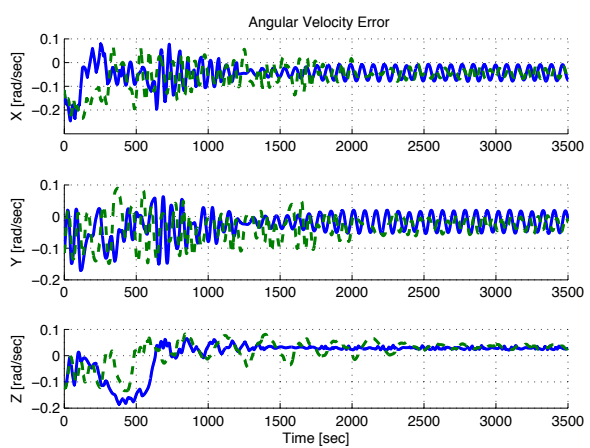

(b) Angular Rate Error
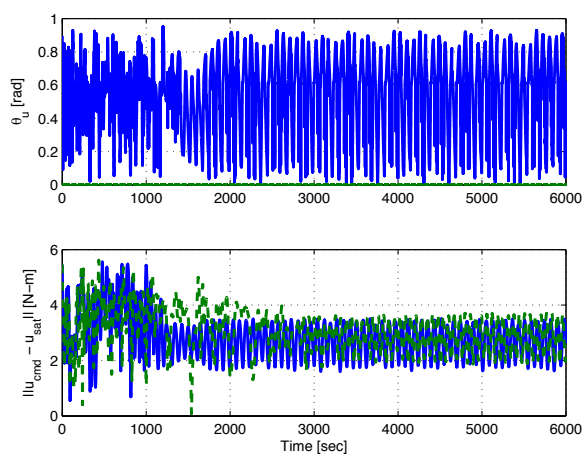

(d) Control Vector Comparison

Figure 14: M2S maneuver about a non-principal body-fixed spin axis with $\left\|\omega_{d}\right\|=0.1 \mathrm{rad} / \mathrm{sec}$ without disturbances. Performance is compared for the VAG-C and the VAG-S algorithms imposing a $0.005 \mathrm{~N}-\mathrm{m}$ saturation limit.

shows that the algorithm fails to reach steady state. As seen in Figure 18, the disturbance estimates diverge, causing the controller to continually command torques to fight a "phantom" disturbance. This problem can be addressed by applying the VAG algorithm to the adaptive disturbance estimates.

\section{Conclusions and Future Research}

In the paper we examined recently developed inertia-free spacecraft attitude control algorithms in presence of actuation torque magnitude limits. For the motion-to-rest controller, the controller gains can always be selected to guarantee the specified torque magnitude limits while the controller is capable of achieving a desired orientation starting from almost all initial orientations and angular rates. For the dynamic motion-to-spin controller, we described an approach to decrease the adaptation rate in the presence of saturation, and we numerically demonstrated the controller's ability to reach steady state for both principalaxis spins and arbitrary axis spins. In addition, the performance degradation of the closed-loop response was studied in presence of constant disturbance torque inputs and actuation torque-magnitude limits. While controller performance can degrade significantly in the presence of disturbances, in many cases graceful and reasonable performance degradation was observed. We have also presented an extremum tracking controller that seeks online closest achievable spin rate about the specified axis while satisfying the imposed torque magnitude constraints. 


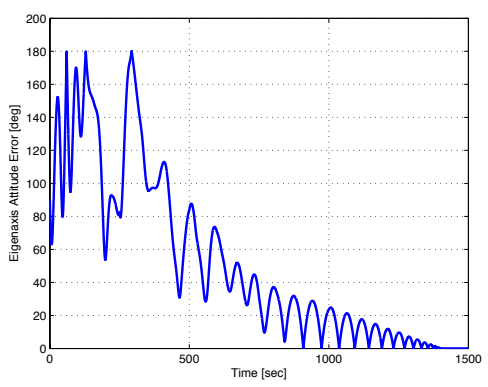

(a) Eigenaxis Attitude Error

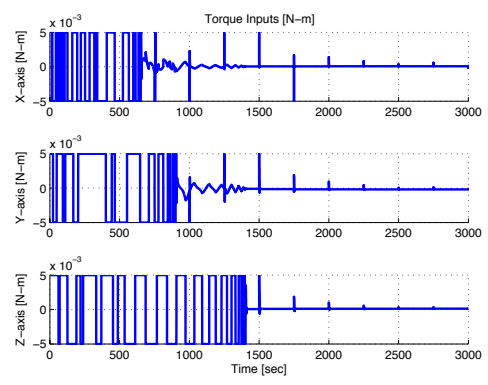

(c) Control Torque
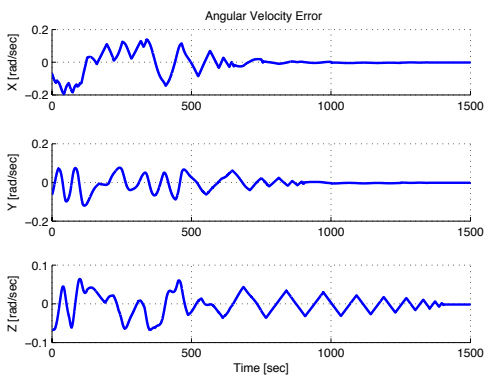

(b) Angular Rate Error

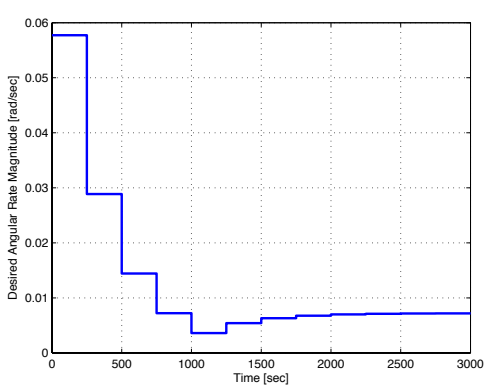

(d) Desired Rate Magnitude

Figure 15: M2S about an arbitrary axis with $\left\|\omega_{d, 0}\right\|=0.1 \mathrm{rad} / \mathrm{sec}$ without disturbances. Performance for Extremum Tracking and VAG-C Imposing a 0.005 N-m saturation limit.

\section{References}

[1] Sanyal, A., Fosbury, A., Chaturvedi, N., and Bernstein, D. S., "Inertia-Free Spacecraft Attitude Tracking with Disturbance Rejection and Almost Global Stabilization," AIAA J. Guid. Contr. Dyn., Vol. 32, pp. 1167-1178, 2009.

[2] Weiss, A., Yang, S., Kolmanovsky, I., and Bernstein, D. S., "Inertia-Free Spacecraft Attitude Control with Reaction-Wheel Actuation," AIAA Guid. Nav. Contr. Conf., Toronto, August 2010, AIAA-20108297-163.

[3] Wie, B., and Barba, P. M., "Quaternion Feedback for Spacecraft Large Angle Maneuvers," AIAA Journal of Guidance, Control, and Dynamics, Vol. 8, pp. 360-365, 1985.

[4] Joshi, S. M., Kelkar, A. G., and Wen, J. T., "Robust attitude stabilization using nonlinear quaternion feedback," IEEE Transactions on Automatic Control, Vol. 40, pp. 1148-1161, 1995.

[5] Ahmed, J., Coppola, V. T., and Bernstein, D. S., "Asymptotic Tracking of Spacecraft Attitude Motion with Inertia Matrix Identification," AIAA Journal of Guidance, Control, and Dynamics, Vol. 21, pp. 684-691, 1998.

[6] Crassidis, J. L., Vadali, S. R., and Markley, F. L., "Optimal Variable-Sructure Control Tracking of Spacecraft Maneuvers," AIAA Journal of Guidance, Control, and Dynamics, Vol. 23, pp. 564-566, 2000.

[7] Bhat, S. P., and Bernstein, D. S., "A Topological Obstruction to Continuous Global Stabilization of Rotational Motion and the Unwinding Phenomenon," Systems and Control Letters, Vol. 39, pp. 63-70, 2000.

[8] Chaturvedi, N. A., Global Dynamics and Stabilization of Rigid Body Attitude Systems, Ph.D. Dissertation, University of Michigan, 2007. 


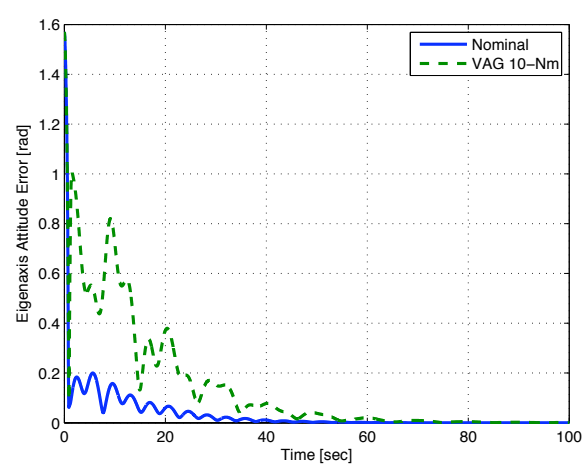

(a) Eigenaxis Attitude Error

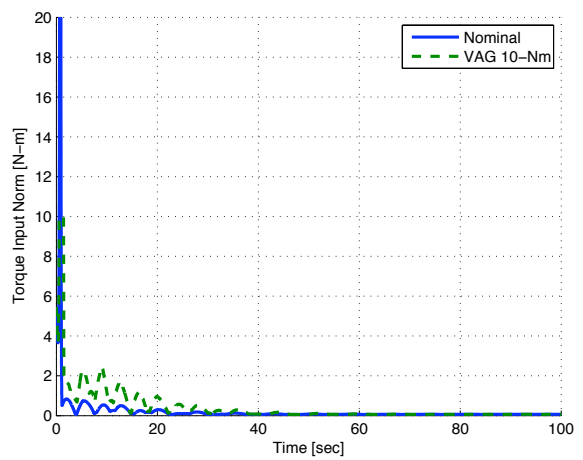

(c) Control Torque Infinity Norm
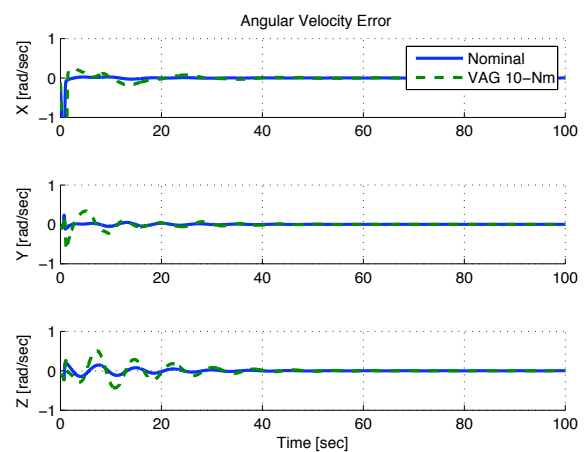

(b) Angular Rate Error
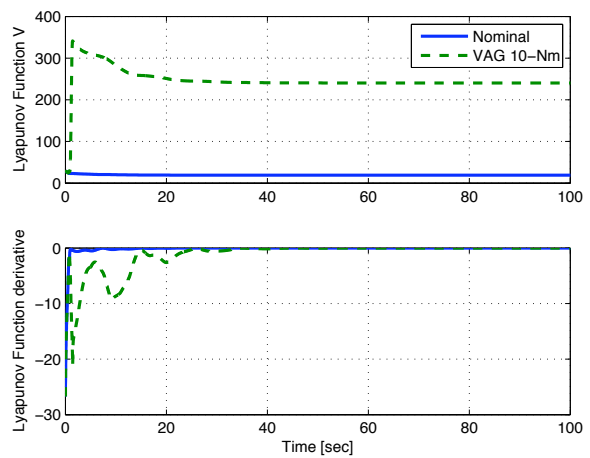

(d) Lyapunov Function and Derivative

Figure 16: Motion to spin maneuver about a non-principal body-fixed spin axis with $\left\|\omega_{d}\right\|=0.1 \mathrm{rad} / \mathrm{sec}$ under a constant disturbance torque. Performance is compared for the unsaturated case and a $10-\mathrm{N}-\mathrm{m}$ saturation limit imposed by VAG-C algorithm.

[9] Sanyal, A. K., and Chaturvedi, N. A., "Almost Global Robust Attitude Tracking Control of Spacecraft in Gravity," Proceedings of the AIAA Guidance, Navigation, and Control Conference, Honolulu, HI, August 2008, AIAA-2008-6979.

[10] Chaturvedi, N. A., and McClamroch, N. H., "Attitude Stabilization of the Inverted 3D Pendulum on TSO(3) with Control Saturation," IEEE Conference on Decision and Control, New Orleans, December 2007.

[11] Chaturvedi, N. A., McClamroch, N. H., and Bernstein, D. S., "Asymptotic Smooth Stabilization of the Inverted 3D Pendulum," IEEE Transactions on Automatic Control, Vol. 54, pp.1204-1215, 2009.

[12] Chaturvedi, N. A., McClamroch, N. H., and Bernstein, D. S., "Stabilization of a 3D Axially Symmetric Pendulum," Automatica, Vol. 44, pp. 2258-2265, 2008.

[13] Arambel, P. O., Manikonda, V., and Mehra, R. K., "Spacecraft Attitude Tracking in the Presence of Input Magnitude Constraints," Proceedings of the American Control Conference, pp. 4082-4086, June 2000 .

[14] Boskovic, J. D., Li, S.-M., and Mehra, R. K., "Robust Adaptive Variable Structure Control of Spacecraft Under Control Input Saturation," AIAA Journal of Guidance, Control, and Dynamics, Vol. 24, pp. 14$22,2001$.

[15] Banga, H., Tahka, M.-J., and Choib, H.-D., "Large Angle Attitude Control of Spacecraft with Actuator Saturation," Control Engineering Practice, Vol. 11, pp. 989-997, 2003. 

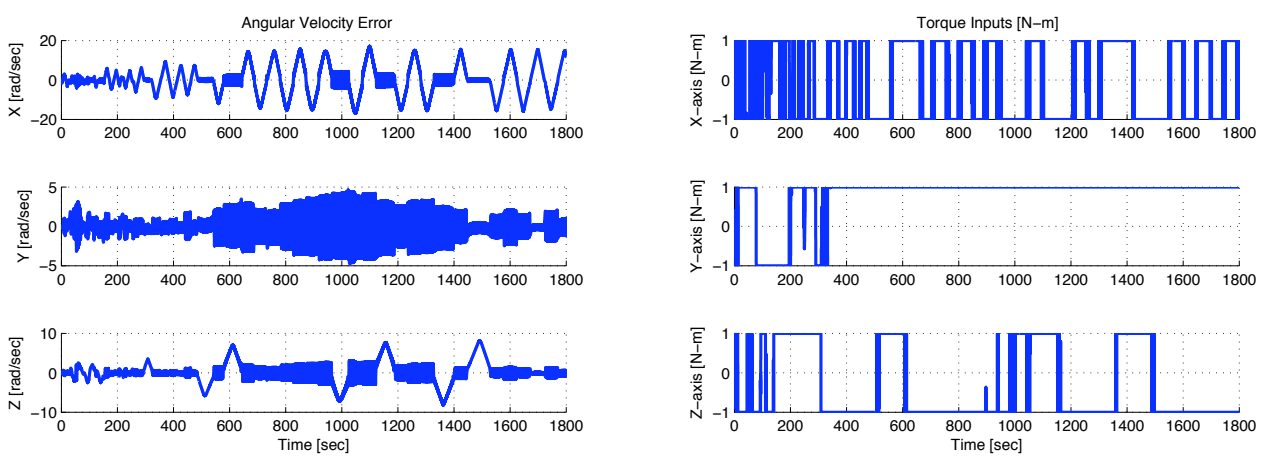

(a) Angular Rate Error

(b) Torque

Figure 17: Motion to spin maneuver about a non-principal body-fixed spin axis with $\left\|\omega_{d}\right\|=0.1 \mathrm{rad} / \mathrm{sec}$ under a constant disturbance torque. A 1-N-m saturation limit was imposed with the VAG-C algorithm.
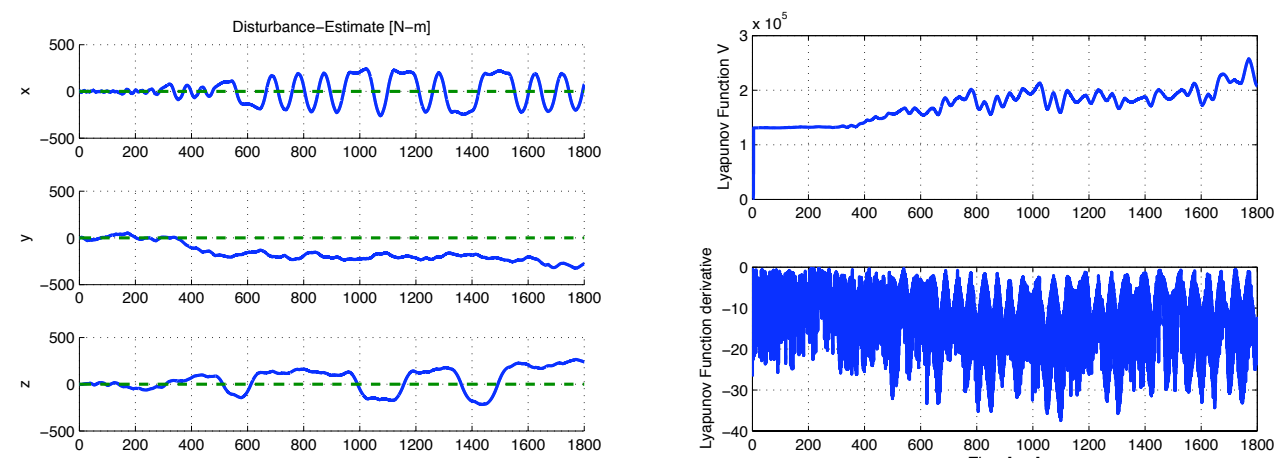

(a) Disturbance Torque Estimate

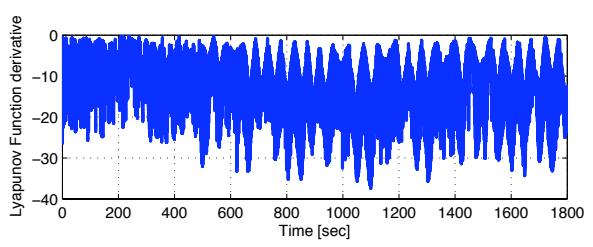

(b) Lyapunov Function and Derivative

Figure 18: Inertia estimates and Lyapunov candidate for the non-principal body-fixed spin axis maneuver with disturbances with 1-N-m saturation limit imposed by the VAG-C algorithm.

[16] Hughes, P. C., Spacecraft Attitude Dynamics, Wiley, 1986; reprinted by Dover, 2008. 